University of Louisville

ThinkIR: The University of Louisville's Institutional Repository

Electronic Theses and Dissertations

8-2015

\title{
Awareness of executive functioning as a diagnostic tool for mild cognitive impairment and Alzheimer's disease.
}

Jeremy Stark Carmasin

University of Louisville

Follow this and additional works at: https://ir.library.louisville.edu/etd

Part of the Clinical Psychology Commons

\section{Recommended Citation}

Carmasin, Jeremy Stark, "Awareness of executive functioning as a diagnostic tool for mild cognitive impairment and Alzheimer's disease." (2015). Electronic Theses and Dissertations. Paper 2260.

https://doi.org/10.18297/etd/2260

This Doctoral Dissertation is brought to you for free and open access by ThinkIR: The University of Louisville's Institutional Repository. It has been accepted for inclusion in Electronic Theses and Dissertations by an authorized administrator of ThinkIR: The University of Louisville's Institutional Repository. This title appears here courtesy of the author, who has retained all other copyrights. For more information, please contact thinkir@louisville.edu. 


\title{
AWARENESS OF EXECUTIVE FUNCTIONING AS A DIAGNOSTIC TOOL FOR MILD COGNITIVE IMPAIRMENT AND ALZHEIMER'S DISEASE
}

\author{
By \\ Jeremy Stark Carmasin \\ B.A., Brandeis University, 2007 \\ M.A., University of Louisville, 2013

\begin{abstract}
A Dissertation
Submitted to the Faculty of the

College of Arts and Sciences of the University of Louisville

in Partial Fulfillment of the Requirements

for the Degree of
\end{abstract}

Doctor of Philosophy in Clinical Psychology

Department of Psychological and Brain Sciences

University of Louisville

Louisville, Kentucky

August 2015 
Copyright 2014 by Jeremy Stark Carmasin

All rights reserved 



\title{
AWARENESS OF EXECUTIVE FUNCTIONING AS A DIAGNOSTIC TOOL FOR MILD COGNITIVE IMPAIRMENT AND ALZHEIMER'S DISEASE
}

\author{
By \\ Jeremy Stark Carmasin \\ B.A., Brandeis University, 2007 \\ M.A., University of Louisville, 2013
}

A Dissertation Approved on

June 26, 2014

by the following Dissertation Committee:

Dissertation Chair

Benjamin Mast, Ph.D.

Suzanne Meeks, Ph.D.

Jay Irby, Ph.D.

John Pani, Ph.D.

Robert Friedland, M.D. 


\title{
DEDICATION
}

This dissertation is dedicated to my grandmothers

\author{
Margaret Stark \\ and \\ Ethel Carmasin
}

who taught me how to age with grace, fortitude, and humor. 


\section{ACKNOWLEDGMENTS}

I would like to foremost thank my mentor, Dr. Benjamin Mast, for his constant empathy, insight, and encouragement throughout my time in the program. Many thanks to my committee members for their support in this project and for the wisdom they have shared in lectures and supervision. I am grateful to my many Louisville friends, especially my geropsychology colleagues Brittney Getz and Jessica Strong, for making sure I never felt alone exploring a new environment or working on a difficult task.

Finally, I would like to thank my parents, Judy and Alan Carmasin, for reminding me that every part of the journey matters. 


\title{
ABSTRACT \\ AWARENESS OF EXECUTIVE FUNCTIONING AS A DIAGNOSTIC TOOL FOR MILD COGNITIVE IMPAIRMENT AND ALZHEIMER'S DISEASE
}

\author{
Jeremy Stark Carmasin
}

June 26, 2014

This study examined the utility of older adults' awareness of their executive functioning abilities to predict future cognitive decline. The recently revised Cognitive Awareness Model (CAM) forms the conceptual background of this approach, and suggests that executive dysfunction disrupts awareness in a manner distinct from episodic memory dysfunction. The study design examined how awareness of executive functioning ability may predict both continuous decline on neuropsychological testing and qualitative change in diagnostic status. This form of prediction was tested using a longitudinal sample $(n=661)$ of older adults with either normal cognition, mild cognitive impairment (MCI), or Alzheimer's disease (AD). Executive functioning awareness was operationalized as Everyday Cognition (ECog) questionnaire discrepancy scores, made up of the difference between informant and subject reports of daily task efficiency. Executive functioning discrepancy scores were shown to have moderate utility at predicting cognitive decline on several measures and modest utility at predicting diagnostic change. However, modest convergent validity and low discriminant validity were observed for executive functioning discrepancy scores in the context of memory discrepancy scores. Future research directions are reviewed, including the need to 
document the time course of early memory awareness deficits vs. early executive functioning awareness deficits. 
TABLE OF CONTENTS

PAGE

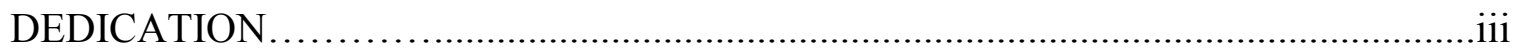

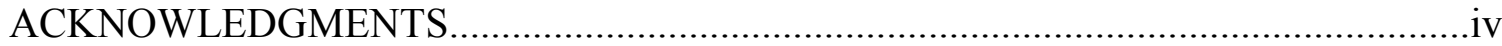

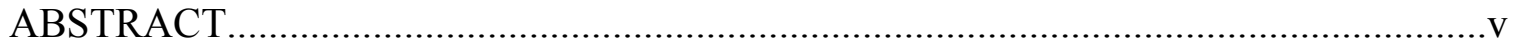

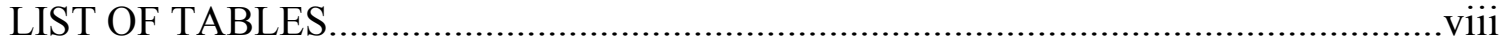

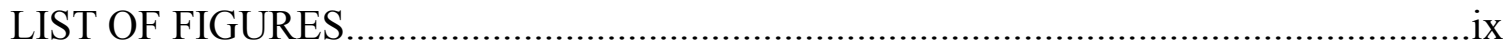

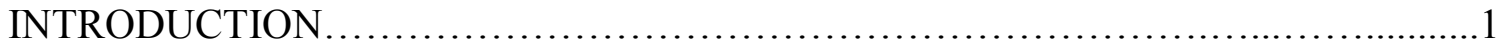

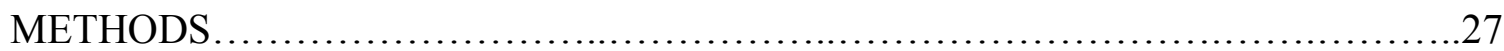

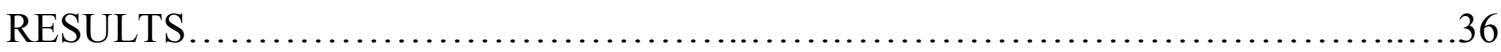

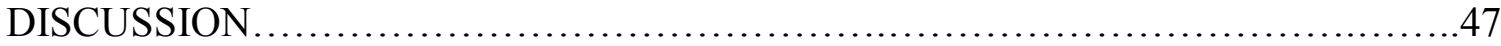

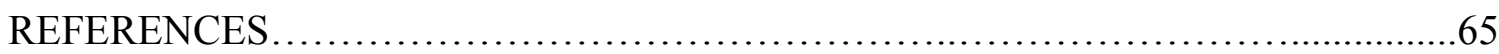

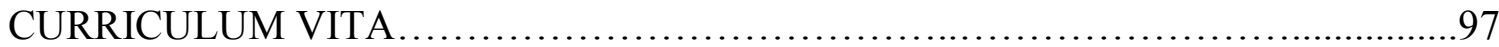




\section{LIST OF TABLES}

TABLE

PAGE

1. Study Sample Characteristics and Correlations with ECog Scales - Normal and MCI Subjects...........74

2. Linear Regression Model of ECog EF Predicting Logical Memory.....................................................75

3. Linear Regression Model of ECog EF Predicting Trail Making Part B …............................................76

4. Linear Regression Model of ECog EF Predicting RAVLT..................................................................

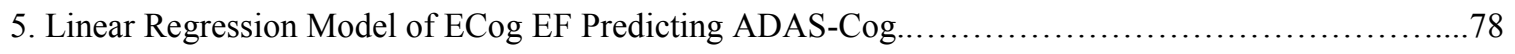

6. Linear Regression Model of ECog EF Predicting Clock Drawing....................................79

7. Logistic Regression Models of ECog EF Predicting Diagnostic Conversion........................80

8. Study Sample Characteristics and Correlations with ECog Scales - All Subjects......................81

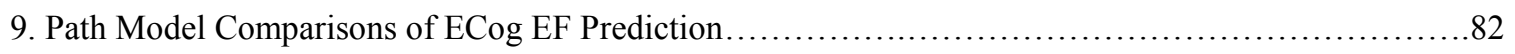

10. Linear Regression Model of ECog Memory Predicting Logical Memory ...............................83

11. Linear Regression Model of ECog Memory Predicting Trail Making Part B ..........................84

12. Linear Regression Model of ECog Memory Predicting RAVLT .................................. 85

13. Linear Regression Model of ECog Memory Predicting ADAS-Cog ................................ 86

14. Linear Regression Model of ECog Memory Predicting Clock Drawing ............................87

15. Logistic Regression Models of ECog Memory Predicting Diagnostic Conversion.........................88

16. Linear and Logistic Regression Models of Simultaneous ECog EF and Memory Prediction............89 


\section{LIST OF FIGURES}

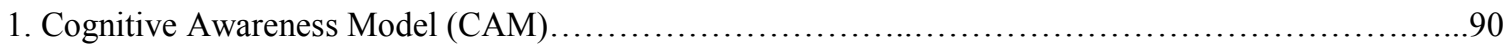

2. Histogram of normal and MCI executive functioning discrepancy scores............................91

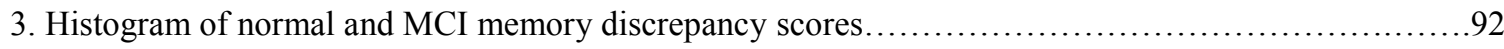

4. Month 12 normal memory model of ECog executive functioning discrepancy prediction........................93

5. Month 12 impaired memory model of ECog executive functioning discrepancy prediction..............94

6. Month 24 normal memory model of ECog executive functioning discrepancy prediction................95

7. Month 24 impaired memory model of ECog executive functioning discrepancy prediction...............96 


\section{INTRODUCTION}

\section{Defining and modeling awareness}

Overview of decline in mild cognitive impairment and Alzheimer's disease.

The prevalence of both mild cognitive impairment (MCI) and Alzheimer's disease (AD) increases with age, with both disorders most frequently diagnosed in people age 65 and older. MCI is a syndrome involving either 1) memory impairment in the context of otherwise normal cognitive functioning, or 2) no memory impairment, but impairment in one or more other cognitive domains. In both MCI permutations, the patient retains normal functional abilities in the context of their age and level of physical disability. An estimated $8-55 \%$ of people with $\mathrm{MCI}$ will convert to AD within five years of receiving an MCI diagnosis (Collie, Maruff, \& Currie, 2002). In research and clinical practice, MCI is often a transition between normal aging and early-stage $\mathrm{AD}$, but is also considered distinct from both. Under the most recently proposed National Institute on Aging Alzheimer's Association (NIA-AA) clinical diagnostic criteria, MCI requires “concern regarding a change in cognition" from a patient, a close informant, or a skilled clinician who has observed the patient. (A patient's own concerns may also be labeled "selfreports" or "subjective complaints.") The patient must also display impairment in memory, executive functioning, visuospatial skills, attention, or language. They may display some reduced efficiency or accuracy at these tasks compared to previous reports. However, a patient must not display substantial impairment in social functioning, 
occupational function, or independent living (e.g. paying bills, cooking, etc.; Albert et al., 2011).

Although some older adults with MCI do not experience further cognitive decline, $\mathrm{AD}$ is explicitly a degenerative illness. Early stage $\mathrm{AD}$ involves the gradual deterioration of cognitive abilities. The earliest symptoms of AD include substantial difficulty with memory and instrumental activities of daily life (e.g. driving, shopping for food), as well as an increase in apathy and confusion in unfamiliar situations. Although the most prominent losses typically involve memory, the disease also affects the domains of language, attention, and executive functioning. Middle and late stage AD patients continue to decline in the above areas, but also show more pronounced behavior changes and confusion. The combination of these declines persisting for several months is considered dementia (Welsh-Bohmer \& Warren, 2006).

Both $\mathrm{MCI}$ and $\mathrm{AD}$ represent difficulty in cognitive domains beyond what is expected in normal aging (Welsh-Bohmer \& Warren, 2006). Earlier diagnosis of these disorders is valuable for research (more opportunities for larger subject samples and longitudinal data collection) and clinical practice (a longer period of time to employ medication, psychotherapy, and behavioral interventions; Bradford, Kunik, Schulz, Williams, \& Singh, 2009). Additionally, earlier diagnosis entails earlier financial benefits for patients and health care providers, such as access to insurance benefits from programs such as Medicare (Weimer \& Sager, 2009). Improving diagnostic accuracy will likely entail examining awareness in each domain known to decline in $\mathrm{MCI}$ and $\mathrm{AD}$, including executive functioning. 
Overview of executive functioning

Executive functioning is a broad term for the regulation and management of other cognitive processes in order to achieve goals. Executive functioning incorporates attention, organization, cognitive flexibility, and error monitoring. Strongly associated with the state of the frontal lobe, it is critical to the planning and execution of actions (Chan, Shum, Toulopoulou, \& Chen, 2008).

A lack of longitudinal data has impeded the study of executive functioning across the life span. However, components of executive functioning appear to decline in a heterogeneous manner with age, e.g. organization may remain intact despite deficits in inhibition. Additionally, diagnosing a deficit as primarily executive requires ruling out other potential contributors, e.g. difficulty in accessing episodic memory (Jurado \& Rosselli, 2007). In sum, it is useful to assess executive functioning in multiple components, as well as directly compare performance on executive measures to those of other cognitive tests.

Historically, it has been challenging to draw specific conclusions from executive functioning measures. Ecological validity is a problem in executive functioning assessment because complex tasks in daily life require a complex series of steps, including goal-setting, prioritization, and inhibition. Successful executive functioning integrates several of these abilities in the service of an objective. Poor performance on a single measure of executive functioning indicates impairment, but does not determine which of the many processes employed between goal identification and completion are impaired (Chan, Shum, Toulopoulou, \& Chen, 2008). 
The relationship between executive functioning and instrumental tasks in daily life is well understood in later stages of cognitive impairment (Goel, Grafman, Tajik, Gana, \& Danto, 1997; Grafman \& Litvan, 1999), but less so in earlier stages such as MCI (Ready, Ott, Grace, \& Cahn-Weiner, 2003). Gathering context through patient and/or informant reports allows executive functioning measures to better highlight impairment at any stage. For example, studies might add context by querying subjects on their success at specific abilities (shopping, driving, paying bills, etc.). Asking several such direct questions as opposed to abstract perceptions of general success at executive tasks should generate more relevant connections between a subject's performance on standardized measures and their success at daily tasks (Farias et al., 2013).

\section{Definitions of awareness in $M C I$ and $A D$}

Asking subjects questions about their daily tasks is one form of gauging their awareness, which may be used as an indicator or predictor of cognitive decline. Some terms describe awareness for a specific cognitive domain, e.g. metamemory: patients' monitoring of their own memory performance and their knowledge of the memory strategies they employ (Clare, 2004b; Frank, Lenderking, Howard, \& Cantillon, 2011; Marková \& Berrios, 2006). Lack of awareness is distinct from concepts such as lack of concern, anosodiaphoria, and indifference, which each indicate a diminished emotional response to decline rather than diminished awareness.

Awareness is the ability to reconcile new information about one's actions and environment with well-established information within one's self-knowledge (Agnew \& Morris, 1998; Mograbi, Brown, \& Morris, 2009). Awareness may be most easily 
understood by examining its absence. Damage to various brain regions, such as the entorhinal cortex and hippocampus in the medial temporal lobe, can disturb cognitive domains such as memory, language, and executive functioning during the insidious progression of AD (Braak \& Braak, 1991). Many patients express a lack of awareness of these deficits (i.e. anosognosia), ranging for overestimating their abilities to explicitly rejecting the presence of decline (McGlynn \& Schacter, 1989). If an older adult can accurately describes their abilities within a cognitive domain ("good," "okay," "poor," etc.), then they have "explicit awareness" about that domain. In contrast, having "implicit awareness" refers to an incomplete state of self-knowledge, wherein affect and/or behavior may change in response to new information, but there is no conscious recognition of the new information. For example, a woman might insist that she has no memory impairment, but also attempt to avoid any memory-dependent tasks (Agnew \& Morris, 1998; Clare et al., 2011).

Awareness should not be conceptualized as an all-or-nothing phenomenon: Patients can be aware of difficulty in some domains but not others, or even inconsistently aware of difficulty within the same domain, e.g. complaints of difficulty with verbal memory but not visual memory (Mograbi, Brown, \& Morris, 2009). Memory deficits alone typically do not account for lack of awareness in AD (Ecklund-Johnson \& Torres, 2005). Rather, awareness in $\mathrm{AD}$ is frequently impaired due to a combination of cognitive difficulties. Episodic memory gradually deteriorates in $\mathrm{AD}$, yet many patients deny cognitive problems even when they are unable to recall any recent events. Anosognosia also impairs a patient's ability to see themselves from the third person (i.e. difficulty 
understanding how other people view them), further hindering their ability to adapt to feedback on their cognitive performance (Salmon et al., 2005).

An improved understanding of awareness in the context of MCI and AD will ultimately increase awareness's viability as a diagnostic tool. Aspects of awareness that decline predictably with observable cognitive changes may be especially useful to study (Clare, Marková, Verhey, \& Kenny, 2005). MCI and AD are currently diagnosed through a combination of patient reports, informant reports, neuropsychological testing, neuroimaging, and/or biomarkers such as blood sampling. Awareness increasingly appears to be a useful tool to distinguish between normal aging, MCI, and early stages of

AD: The level of awareness deficit varies within both MCI and early-stage AD (Cosentino, Metcalfe, Butterfield, \& Stern, 2007; Roberts, Clare, \& Woods, 2009). This variance suggests that changes in awareness may reveal cognitive decline.

\section{Subject/informant discrepancy scores}

Awareness is a complex concept. Cognitive complaints and anosognosia have been operationalized in studies of $\mathrm{MCI}$ and $\mathrm{AD}$ in numerous ways, including subjective ratings from a subject or informant, and discrepancy scores between subject and informant ratings. Awareness is frequently gauged using a subject or informant's answers to a questionnaire, which may be in a Yes/No, Likert scale, or other format. This approach is typically combined with at least one objective measure of performance, such as the subject's score on a neuropsychological test. Some awareness questionnaires have also been operationalized into discrepancy ratings: an informant (typically a family member or close friend, and often a caregiver if the subject has a diagnosis of AD) 
completes the same questionnaire as the subject. Such scores are frequently calculated through simple subtraction, such as Informant Score - Subject Score. The result can then be interpreted on a continuum, such as large, positive scores meaning the informants rated much more impairment than the subjects. Discrepancy scores assume that an informant provides an accurate and valid rating of the subject's performance, and that any instances where the subject reports better functioning than the informant represents an overestimation of the subject's ability (Clare et al., 2005). For this study, a "significant discrepancy" or "greater discrepancy" indicates the subject significantly overestimated ability compared to the informant unless otherwise noted.

The discrepancy method has shown moderate effectiveness to date, though reliability is a frequent concern. Specifically, individual differences in informant personality, as well as differences in the nature of the relationship between subject and informant, appear to sway informant ratings (Clare, 2004a; Green, Goldstein, Sirockman, \& Green, 1993). However, unreliable informant reports may be a result of measures with very broad questions, e.g. "Does your spouse/parent/friend/etc. have trouble with their memory?" It may be unreasonable to expect informants to know what "normal" memory means for an older adult. "Normal" must be contextualized by a variety of factors, such as level of education and medical history. Additionally, study informants may be biased by stereotypes and anecdotal evidence regarding other older adults' cognitive abilities. Measurement via direct, unambiguous functional questions should improve the accuracy of the discrepancy method. Moreover, questionnaires could ask for a comparison to a subject's performance several years in the past, rather than asking (explicitly or implicitly) to make a comparison to the subject's peer group. This method allows the 
respondent to serve as their own control in a salient manner. For example, the respondent can indicate that, although the subject has a poor memory for directions, this does not represent a decline from the previous decade - the subject has always had a poor memory for directions (Farias et al., 2008; Schinka, 2010).

\section{Awareness assessment requires integrating more than memory}

As discussed earlier, memory is a critical piece of dementia-related decline, and is therefore intertwined with awareness of said decline. In a recent review, the overt expression of awareness of impairment was found to be very low at late stages of dementia (Clare, 2010). Another review found that larger memory awareness discrepancy scores between patients and caregivers have been observed in later stages of impairment. However, memory deficits alone did not account for lack of awareness in milder stages of $\mathrm{AD}$, as observed in studies of patients with amnesic deficits who retained high awareness of memory impairment (Ecklund-Johnson \& Torres, 2005). A focus on anosognosia for memory to the exclusion of other cognitive domains may be part of the problem: Historically, anosognosia for memory has not consistently been related to objective memory test performance in MCI and mild AD (Mograbi et al., 2009).

Memory tests that exclusively involve recall and recognition (e.g. memorization of a word list followed by immediate and delayed retrieval trials) may not be sufficiently effortful to distinguish between normal and impaired memory. For example, a traditional recognition task would involve a prompt such as "Say Yes if you heard this word earlier." A more effortful task could state "Say Yes only if this item was presented to you as a picture rather than as text." In a study of retrieval monitoring and anosognosia, healthy 
older adults and mild $\mathrm{AD}$ patients each completed an anosognosia questionnaire and were then shown several stimuli, some presented as text and others presented as pictures. Both healthy controls and mild AD patients appeared able to distinguish between studied and not-studied items. However, the mild AD patients displayed difficulty recalling the presentation format (in the above case, text vs. pictures). Among the mild AD patients, greater anosognosia appears correlated with greater difficulty in this comparatively complex task but not in the simple recognition task (Gallo, Chen, Wiseman, Schacter, \& Budson, 2007).

The combination of poor effortful task ability and poor memory self-knowledge may be interpreted as dysfunction within the frontal lobe. This region is critical in making accurate evaluations of one's own memory (Pannu \& Kaszniak, 2005), and dysfunction within the frontal lobe is associated with deficits in both executive functioning and self-monitoring (Antoine, Antoine, Guermonprez, \& Frigard, 2004; Pia \& Conway, 2008). As a result, patients who face an effortful task and have impaired executive functioning skills (e.g. organization, inhibition), will have poor performance that is substantially more negative than their self-report. In contrast, the lower-effort word recognition tasks may have posed a similar challenge to patients both with and without frontal lobe damage. In the 2007 Gallo et al. study, the recognition task was made more effortful by requiring subjects to engage processes beyond a sense of familiarity (the context-free sensation that something has been encountered in the past). AD patients appear capable of using familiarity to aid recognition into moderate or later stages of the disorder (Pannu \& Kaszniak, 2005; Souchay, 2007). Studies should therefore not rely 
solely on familiarity measures when attempting to distinguish between awareness in healthy cognition, MCI, and mild AD.

Both executive and metamemory dysfunction are commonly linked to dysfunction in the frontal lobe, particularly the prefrontal cortex (McGlynn \& Schacter, 1989; Pannu \& Kaszniak, 2005). The frontal, medial parietal, and lateral-temporoparietal regions are part of the neural pathways engaged in healthy adults when retrieving memories or attempting to understand other people's perspectives, known as the default mode network (Buckner, Andrews-Hanna, \& Schacter, 2008). This network has shown reduced activity among $\mathrm{AD}$ patients, suggesting that there may be a link between dysfunction in these awareness-relevant areas and AD-related cognitive decline (Zamboni \& Wilcock, 2011).

In a meta-analysis of studies of memory awareness in MCI and dementia patients, there were significantly more subjective memory complaints among dementia patients than among MCI patients. A majority of the examined studies contained simple questions asking if subjects had trouble with memory. High sensitivity indicated that the measure typically predicted a "true" (as diagnosed by physician) dementia diagnosis, while high specificity indicated the measure accurately rejected a "false" dementia diagnosis. The pooled sensitivity (aligning with a physician's diagnosis of dementia) of complaints in dementia was $43.0 \%$, while specificity (aligning with a physician's diagnosis of no dementia) was $85.8 \%$. The pooled dementia positive predictive validity was $18.8 \%$ and pooled negative predictive validity was $93.7 \%$. The pooled sensitivity of complaints in MCI was $37.4 \%$, while specificity was $86.9 \%$. The pooled MCI positive predictive validity was $31.4 \%$ and pooled negative predictive validity was $86.9 \%$. The findings suggest that, although the absence of memory complaints may assist in ruling out a 
diagnosis of MCI or dementia, memory complaints alone are not a useful method for detecting these disorders (Mitchell, 2008). Awareness of memory measured in tandem with awareness of executive functioning may provide a more complete profile of cognitive loss.

\section{Limitations of summarizing awareness in a single domain}

Given the association between AD progression and memory decline, memory impairment is studied especially frequently within MCI and AD awareness research (Clare et al., 2011; Roberts et al., 2009). In earlier research designs, an older adult's view on their memory ability was often gauged by a brief interview or a handful of multiple choice responses. As detailed below, this approach may mask subtleties inherent in cognitive decline. Furthermore, narrow study designs may exclude crucial information from other cognitive domains.

In one such design, AD patients' medical records were reviewed, including the clinical interviews, labs, and neuropsychological evaluations. Awareness was assessed through the single question, "Do you have a memory problem that makes your life more difficult or complicated?” In logistic regression analysis with dichotomized age $(>70$ years) and education ( $>12$ years), executive functioning was a significant predictor of awareness $(R=0.19, p=0.003)$. The authors concluded that lack of awareness is related to declines in executive functioning and general cognition (Lopez, Becker, Somsak, Dew, \& DeKosky, 1994).

Although the association between awareness and executive functioning is promising, this methodology makes it difficult to examine the mechanisms of awareness. 
For example, there is no way to know where the error in self-analysis occurred, i.e. a failure to update long-term self-knowledge vs. a failure to compare recent poor performance to long-term self-knowledge (Morris \& Mograbi, 2013). Not only was selfreport limited to a single domain (memory), but also limited to a single question. This approach demands that patients summarize their views on their memories into a single statement, rather than allowing the nuance of rating memory higher in some settings than others (e.g. memory for dates as higher than memory for conversations). Overall, awareness was operationalized in a simplistic fashion within the study design, casting doubt on whether or not this form of awareness can distinguish between stages of AD.

In a later study, 3,673 community-dwelling older adults were followed for 10 years (one baseline visit and then three additional visits) through repeated administration of a 20-item word recall task, an activities of daily living (ADL) questionnaire, and a 7item memory screening. Subjects were considered to have memory complaints if they rated their memory as "fair, poor, or very poor" on a single question: They were asked to compare their memory to peers of their age. Subjects were classified as either cognitively normal or MCI based on responses to the above measures at a baseline visit. Based on longitudinal analysis, memory complaints did not significantly predict change on the above measures over 10 years. The authors concluded that such complaints could not distinguish normal cognition from MCI (Purser, Fillenbaum, \& Wallace, 2006).

This study contains methodological concerns that cloud the authors' conclusion. For example, the sampling of memory complaints is extremely brief. In contrast to the more detailed sampling of ADLs, there is no data on what specific memory deficits subjects believe they possess. For example, a subject might complain of memory 
difficulties in paying bills that are independent of her deficits in word recall ability. The lack of meaningful overlap between self-report and dependent variable violates a major assumption of awareness questionnaires; that any tasks compared to questionnaire ratings should allow a valid representation of a subject's self-report ratings (Clare et al., 2005). Providing additional and more detailed items as part of the questionnaire would help correct this problem. Furthermore, the authors were unclear if the word recall task involved multiple trials, or a comparison of immediate and delayed administrations. Comparing memory complaints to a only a brief screener and single word recall task carries the risk of discounting not only other cognitive domains that may affect memory awareness (e.g. executive function), but also other measures of memory and verbal ability.

\section{The Cognitive Awareness Model (CAM) and multidimensional anosognosia}

Analysis of the above studies suggests that awareness cannot be summarized in a single question or a single domain. Although memory is crucial to awareness, it appears to be intertwined with other domains in a manner that is only partially understood. In 1998, Agnew and Morris proposed a nuanced model of memory encoding and selfawareness. In this model, all adults possess a personal database of semantic memory (learned facts and general knowledge) that is continuously updated through episodic memory (an adult's recall of events from their own life). By providing continuity, this store of memory allows an individual to both review past information and anticipate future self-relevant events. In people with normal awareness, new input of episodic memory that is consistent with the personal database will not produce any changes. 
However, new input that is inconsistent with the personal database will rewrite part of the database.

The likelihood of a rewrite depends upon both the frequency and severity of the new information. For example, if an adult with normal awareness misplaces his car keys one morning, this will not affect his personal database for very long: New information (e.g. having an uneventful and efficient commute and workday) will soon be encoded to enforce his understanding that his memory is generally fine. However, if he misplaces his keys for five consecutive days, this will update his personal database with a message like: "My memory seems problematic." He would also receive this update if, on the same day he misplaced his keys, he had trouble finding the way to his office and could not recall the name of his assistant. In either case, this message resulting from multiple failures is encoded into an abstract self-concept, rather than a more direct framing such as "I am having great difficulty finding my keys" (Agnew \& Morris, 1998).

The above model was revised several times in order to better explain the heterogeneous presentation of anosognosia. The latest version is known as the Cognitive Awareness Model (CAM). The 2013 CAM presents a hierarchical ordering of memory consolidation, beginning with sensory input (auditory, visual, and/or motor) and ending with metacognitive output and regulated behavior and/or affect (see Figure 1). The new CAM highlights executive functioning as a mid-level process in the hierarchy via a system dubbed Cognitive Comparator Mechanisms (CCMs). In a healthy system, CCMs will identify new self-relevant errors before they reach metacognitive awareness, behavior, or affect. This allows an individual to proceed with new thoughts and actions that are influenced by the knowledge that they recently made an error, e.g. "I've missed 
turns three times now on this road, maybe I should check a map for this neighborhood" (Morris \& Mograbi, 2013). Using this model, the authors proposed three forms of awareness deficit:

1) Mnemonic anosognosia, wherein a patient compares a cognitive error to the personal database, but the personal database is not updated. This may result in an overt acknowledgement soon after the error is made. In the case of the office worker described above, this could lead to the semantic construction of "I must have some memory trouble" immediately after forgetting the name of his assistant. However, the information quickly degrades and is not consolidated. It is not preserved in long-term memory and will therefore not affect metacognition hours later. When the personal database is rarely or never updated for an extended period of time, patients may view their cognitive abilities to be as good as they were years or even decades earlier. In these cases, episodic details of the distant past are the only salient details available. Patients' self-concepts are thus "petrified," referring repeatedly to the same outdated sources of self-relevant information (Klein \& Lax, 2010; Mograbi et al., 2009).

2) Executive anosognosia, wherein information flow to or from the CCMs is disrupted and a patient does not successfully monitor errors as they are made. The recent poor performance is not adequately processed through the personal database. Therefore, the patient does not incorporate the meaning and consequences of the failure into their self-knowledge. The patient's error is either not acknowledged, or is acknowledged in its immediate aftermath without regard to the consequences. Upon forgetting the assistant's name, the patient might 
erroneously think "There is no problem. I do not need to know his name to do my job." This contrasts with the genuine but transient concern present in mnemonic anosognosia. Without a direct connection to compare task successes and failures, performance monitoring becomes much less efficient.

3) Primary anosognosia, wherein there is a dysfunction in the Metacognitive Awareness System (MAS), which exists at the top of the CAM hierarchy. This form of disorder, unlike mnemonic and executive anosognosia, may involve successful consolidation of memory into the personal database and successful error comparison. However, this persistent information will not reach conscious awareness. The CAM emphasizes that no single system is considered an awareness center, which underscores how implicit awareness of decline may still emerge for an older adult with primary anosognosia (Morris \& Mograbi, 2013). For example, the office worker's implicit awareness of forgetting the assistant's name would still influence his behavior and affect. He might feel a sense of embarrassment whenever he encounters the assistant, or might place a staff directory prominently on his desk. However, if another colleague noticed this behavior and asked him if he was worried about forgetting names, he would not describe any difficulty with recall.

In sum, the CAM presents awareness as both implicit and explicit, and advocates that implicit forms of awareness are more resistant to cognitive decline than their explicit counterparts (Agnew \& Morris, 1998; Mograbi et al., 2009; Morris \& Mograbi, 2013). 


\section{Refining multidimensional awareness measurement}

Just as the CAM has become more nuanced with revision, awareness

measurement has evolved beyond very brief sampling of subjective memory complaints. The following studies gather awareness data in more detailed and innovative ways than previously discussed designs. They also lay the foundation for future studies to broaden awareness measurement beyond the domain of memory.

A cross-sectional study examined the 1998 Agnew and Morris model (Ansell \& Bucks, 2006), recruiting 18 older adults with mild AD and 18 healthy controls. Subjects were asked to learn a 10-item word list over three trials and then complete a 20-minute delayed recall trial. They were then asked to predict their future level of recall after the third trial and after the delayed recall on a 5-point judgment scale. As expected, the healthy control group was significantly more accurate at estimating their ability than the mild AD group $(F(1,34)=15.11, p<0.01)$. However, the AD patients gave a significantly lower estimate of their memory ability on the 5-point scale between the first and third trials $(z(N=18)=-2.17, p=0.03)$, suggesting that these patients revised predictions downward between the back-to-back trials. A trend-level difference also emerged between the pre-testing and delay phases $(z(N=18)=-1.94, \mathrm{p}=0.05)$, but not between the third trial and the delayed recall trial. The overt but fleeting acknowledgement of memory problems reflects mnemonic anosognosia. In contrast, the healthy controls did not show significant differences between trials. Additionally, global memory awareness was assessed at the beginning of the testing session, after the third trial and after the delay trial with a six-item series of yes/no questions. The AD group rated their global memory less favorably between the beginning of the testing session and 
the end of the third trial $(t(17)=-2.67, p=0.02)$, but not between the pre-testing phase and the delay phase or the third trial and the delay phase. The findings suggest that by incorporating working memory, predictions among $\mathrm{AD}$ patients can improve between brief estimations.

The ability to revise predictions immediately between trials suggests transient awareness in early stage $\mathrm{AD}$, even though said awareness was shown to be inferior to that of healthy controls. Following the 1998 Agnew and Morris model, these AD patients appeared to show mnemonic but not executive anosognosia. Specifically, they displayed brief awareness that memory was worse than the personal database indicated at the beginning of the test. However, they could not update the database with this new information. The trend-level finding that estimation accuracy did not return to baseline levels after a 20-minute delay suggests a weaker relationship between episodic memory and the personal database than the 1998 model proposed (Ansell \& Bucks, 2006). However, a similar methodology using a 60-minute delay instead of a 20-minute delay found that estimations eventually returned to baseline levels. The CAM includes working memory information at the bottom of the hierarchy, where it directly follows the input of sensory information. Thus, differences in minutes could substantially affect results. This finding ultimately supports the 2013 CAM framework, wherein the direct paths from working and episodic memory to semantic memory (the personal database) are disrupted in mild $\mathrm{AD}$, suggestive of mnemonic anosognosia (Stewart, McGeown, Shanks, \& Venneri, 2010).

Another cross-sectional study compared 92 AD patients and 92 case-matched healthy controls. Awareness was examined via three quantitative methods: Experimenter 
Rating Scale (clinicians rating the AD patients' impairment on a 4-point scale), Subjective Rating Discrepancy (both the patient and an informant complete a 42-item questionnaire on memory ability in the patient's everyday life), and Objective Judgment Discrepancy (estimating subjects' accuracy after completing measures of verbal, visual and working memory). Objective Judgment was operationalized with the final score computing the difference between the patient's expected performance ("How many items did you recall on the test you just completed?") and their actual performance on the Rey Auditory Verbal Learning Test (RAVLT). The RAVLT includes five trials of recalling List A, followed by the distractor List B, followed by recalling List A again on immediate and delayed recall trials. Therefore, a subject has multiple opportunities to produce false positive words if they incorrectly verbalize items from the distractor List B. Both Subjective Ratings $(t=8.2, p<0.001)$ and Objective Judgment Discrepancy $(t=$ $15.2, p<0.001)$ indicated more anosognosia among the AD patients than the normal controls. Within the Objective Judgment Discrepancy measures, greater anosognosia (i.e. lower postdiction accuracy) was associated with more errors of intrusion, including immediate false positives $(r=0.47, p<0.001)$ and delayed false positives $(r=0.52, p<$ 0.001; Hannesdottir \& Morris, 2007). Objective Judgment Discrepancy is based on inthe-moment test performance, while the Experimenter Rating Scale and Subjective Rating Discrepancy integrate memory across a variety of settings and over a much longer period of time. As a result, the authors suggest that AD patients in this study may experience mnemonic anosognosia and/or executive anosognosia regarding specific instances of memory use, although this design does not directly distinguish between the two. In contrast, a loss of metacognition (primary anosognosia) may interfere with long- 
term and broad self-knowledge (Hannesdottir \& Morris, 2007). Notably, the three quantitative methods were not significantly related to each other via Pearson correlations, which suggests awareness is a multidimensional construct. Unfortunately, this study included both early and late stages of $\mathrm{AD}$, which are known to have substantially different levels of memory ability and lack of awareness (Welsh-Bohmer \& Warren, 2006). Splitting the AD group into early and late stages could have resolved some confusion regarding primary anosognosia, which is thought to occur more frequently in the later stages of the disease (Agnew \& Morris, 1998).

Another cross-sectional study drew distinctions between executive and primary anosognosia. Eighteen older adult early stage AD patients and 18 normal controls each viewed a sequence of line drawings of common objects and were then presented with a series of word pairs. Subjects had to determine which word in each pair was previously viewed as a line drawing, and also rate the confidence of each guess on a 1-5 scale. After this session was completed, subjects and informants completed the Anosognosia Questionnaire - Dementia (AQD). Early stage AD patients were less likely than controls to express high-confidence in their word guesses $(t=3.10, p=0.004)$. The AD patients also underestimated everyday problems compared to their informants' reports $(t=2.96, p$ $=0.009)$. However, among self-ratings of everyday memory problems, AD patients indicated they experienced more impairment than controls $(t=2.03, p=0.050)$. Greater anosognosia discrepancy on the AQD was correlated with lower recollection accuracy $(r$ $=-0.55, p=0.020$ ) but not metamemory. The authors argued that, because more basic aspects of metamemory are spared in mild $\mathrm{AD}$, these patients may use familiarity more often in their daily judgments than detailed recollection. This could result in inaccurate 
judgments, which are unlikely to be updated to their long-term personal knowledge (Gallo, Cramer, Wong, \& Bennett, 2012).

Similar to the CAM, these findings suggest that short-term comparison (absent in executive anosognosia) is distinct from long-term metacognition (absent in primary anosognosia) (Morris \& Mograbi, 2013). Success in long-term metacognition could cause informants to believe a patient does not have serious deficits in self-awareness. Informants may not recognize that a patient with intact metacognition (no primary anosognosia) may still suffer from mnemonic or executive anosognosia. This could delay a patient's presentation to research and treatment. This also reinforces how both patients and informants in future awareness studies should be queried on both multiple cognitive domains and multiple types of impairment within those domains. This study method might be enhanced through a longitudinal design, given preliminary evidence that mnemonic or executive anosognosia may precede primary anosognosia in AD.

The above studies incorporated elements of objective performance into their conceptualization of awareness in novel ways. Study components dependent on the judgment of patients or informants were generally examined in sufficient detail. Together, these findings suggest that preferred cognitive pathways used by healthy older adults gradually become unusable as cognition declines in AD. Additionally, they demonstrated how implicit awareness may emerge in the context of all three CAM forms of anosognosia: mnemonic, executive, and primary. 


\section{Executive functioning awareness, memory in context, and longitudinal prediction}

The preliminary evidence suggests that executive and/or mnemonic anosognosia emerges before primary anosognosia among AD patients (Agnew \& Morris, 1998; Gallo et al., 2012; Hannesdottir \& Morris, 2007). Therefore, it may be most useful to examine their associated components of the CAM in order to detect changes between normal cognition and MCI, as well as between MCI and AD. Both mnemonic and executive anosognosia rely on systems in the middle of the CAM hierarchy. Executive functioning and its associated CAM systems, CCMs and the personal database, appear underexplored in comparison to the episodic and autobiographic memory systems at the core of mnemonic anosognosia (Clare, 2010). For example, a single verbal memory method is frequently the only measure of objective performance, most often list learning and recall (Ansell \& Bucks, 2006; Mimura \& Yano, 2006; Purser et al., 2006). As the field moves toward more nuanced measurement of awareness, it is necessary to compare memory awareness to awareness of other cognitive domains. As discussed below, a shift toward studying the information flow of the personal database and CCMs may enhance the diagnostic utility of awareness.

Several studies show support for executive functioning components as relevant to anosognosia, either through distinctions in testing (Gallo et al., 2012) or neurobiological differences (Amariglio et al., 2012; Johnson et al., 2007). Many studies also suggest that awareness should be interpreted as a multidimensional construct (Ansell \& Bucks, 2006; Hannesdottir \& Morris, 2007; Mimura \& Yano, 2006). Several relate awareness to known symptoms of MCI and AD, including the potential to broadly identify subjects at greater risk of converting from healthy cognition to impairment (Amariglio et al., 2012; 
Johnson et al., 2007; Saykin et al., 2006). However, most studies do not provide evidence for awareness measures to identify the more subtle cognitive decline between MCI and $\mathrm{AD}$, or between normal aging and MCI. There is also very limited use of longitudinal data, which has been shown as important in establishing relationships between memory complaints and awareness in MCI (Reid \& Maclullich, 2006).

In a recent review of $\mathrm{MCI}$ and mild $\mathrm{AD}$ self-report literature, executive functioning abilities were moderately correlated with other neuropsychological tests (Frank et al., 2011). Informant reports of executive functioning change on the Frontal Systems Behavior Scale (FrSBe) have been positively correlated with increases in other cognitive difficulties among older adults with MCI. However, the authors noted a lack of information on the relationship between executive functioning ability and patient selfreport on said ability. Furthermore, at the time of writing, few measures were available to assess patients' awareness of their executive functioning. The authors noted that selfreport appears to remain a reliable and valid method of evaluating cognitive status until moderate stage AD. However, the preserved domains between healthy cognition and moderate AD vary between patients (e.g. some patients had good executive functioning insight but poor memory insight). $\mathrm{MCI}$ and mild $\mathrm{AD}$ may therefore be the most informative stages of disorder to obtain patient feedback.

Awareness measurement adds a new layer of complexity to the already challenging task of assessing executive functioning in a laboratory setting. Although research into executive functioning awareness is limited, studies have operationalized this awareness into several forms, from success at daily activities (Ott et al., 1996; Suchy, Kraybill, \& Franchow, 2011) to finance management (Van Wielingen, Tuokko, Cramer, 
Mateer, \& Hultsch, 2004) to error monitoring (Amanzio et al., 2013; Mathalon et al., 2003). A common theme among such studies is that both executive functioning performance and awareness deteriorate between healthy aging and early stages of dementia.

In a study of awareness among normal control elders and AD patients, subjects completed the Dementia Rating Scale (DRS), a sensitive measure of cognitive functioning split into five subtests. Before each subtest, subjects were provided general instructions on what they were about to complete, and asked if they thought they would succeed (measured dichotomously). Unlike normal controls, AD patients significantly overestimated performance on their overall predictions $(t>6 ; d f=62 ; p<0.001)$. Among all subtests, the strongest correlation was between the Initiation performance score and the Overall awareness prediction $(r=-0.55, p<0.001)$. Notably, the awareness of Memory prediction was not correlated with any performance scores except for Initiation $(r=-0.26, p<0.05)$. This suggests that, compared to other cognitive domains, executive functioning performance may have an exclusive relationship to certain changes in memory awareness (Antoine, Nandrino, \& Billiet, 2013).

In suggesting that overt memory awareness is correlated with executive functioning performance but not memory performance, the 2013 Antoine et al. study reinforces how the comparison mechanisms within the CAM are critical to healthy (complete) metacognition. A breakdown within the CCMs (executive anosognosia) may be a precursor to more serious deficits in metacognition (primary anosognosia). Expanding the study to include an MCI population could help build a timeline of how anosognosia develops with cognitive decline. This design serves as a useful foundation 
for future studies to integrate executive functioning and memory awareness. The overall method could be enhanced with a larger sample, longitudinal observation, and ratings of specific skills within the domains of memory and executive functioning.

Research questions and hypotheses:

The present study examined the relationship between executive functioning awareness and cognitive performance (general, memory, and executive) over time. Within the CAM, a reduced awareness of executive functioning ability (e.g. organization, dividing attention) suggests $\mathrm{CCM}$ dysfunction due to a lack of comparison between new incoming information and the personal database of extant knowledge (Morris \& Mograbi, 2013). Greater dysfunction in this system indicates less frequent updates to the database, and the patient thus relies on increasingly outdated information. As updates become less frequent, both metacognitive output and behavior/affect regulation are likely to decrease. Therefore, unawareness of executive dysfunction may predict future cognition decline, including disease progression toward MCI and/or AD.

The present study addressed the following research questions:

1) Can older adults' executive functioning awareness serve as a predictor of future cognitive decline? Based on previous cross-sectional designs, executive functioning awareness appears positively correlated with cognitive abilities in multiple domains. It was hypothesized that lower executive functioning awareness (as compared to informant rating) at a baseline visit would predict lower scores on measures of executive functioning and memory performance at 12-month and 24-month follow-up. 
2) Can older adults' executive functioning awareness serve as a predictor of diagnostic change? It was hypothesized that lower executive functioning awareness (as compared to informant rating) at a baseline visit would yield a greater rate of diagnostic conversion from normal cognition toward MCI and $\mathrm{AD}$ at 12-month and 24-month follow-up.

3) Does older adults' objective memory performance moderate the relationship between executive functioning awareness and future cognitive decline? Based on the 2013 CAM, dysfunction in episodic memory can hinder the flow of information into the executive system (CCMs) that compares new errors to existing self-relevant information. It was hypothesized that poorer memory performance at the baseline visit would increase the predictive ability of executive functioning awareness (e.g. the CCMs would be less effective when items lower in the hierarchy were impaired), yielding lower scores on both measures of executive functioning and memory performance at 12-month and 24-month follow-up. 


\section{METHODS}

\section{Subjects}

The data for this study was drawn from the Alzheimer's Disease Neuroimaging Initiative (ADNI) database, which includes English and/or Spanish speaking older adults from 55 sites across the United States and Canada. Subjects included 661 older adults (enrollment ages 55-90). At baseline, each received a diagnosis of normal cognition $(\mathrm{n}=$ 173), MCI $(n=410)$, or mild AD $(n=78)$. All subjects were paired with a study partner:

a friend or family member with at least 10 hours of weekly contact and who accompanied the subject to each ADNI visit. Each subject completed a neuropsychological testing battery, and each subject and a matched study partner completed the Everyday Cognition measure (see Measures section, below) at a baseline visit and at 12-month follow-up. Of these $661 \mathrm{subject/study} \mathrm{partner} \mathrm{pairs,} 247$ also completed these measures at 24-month follow-up.

To qualify as an ADNI normal control (also known as “cognitively normal”), subjects must have had no subjective memory complaints (excepting mild declines associated solely with age). Normal controls required a mini-mental state examination (MMSE) score of 24-30 and a Clinical Dementia Rating (CDR) of 0. Cognitively Normal subjects must have also achieved one of the following scores on the Logical Memory Delayed Recall test: a) $\geq 9$ for subjects with $16+$ years of education, b) $\geq 5$ for subjects with 8-15 years of education, or c) $\geq 3$ for subjects with $0-7$ years of education. 
To qualify as either an ADNI MCI or AD patient, subjects must have had at least one subjective memory concern, which may have emerged from the patient, the study partner, or a clinician. MCI patients required an MMSE of 24-30 and a CDR of 0.5. MCI patients must have achieved one of the following scores on Logical Memory Delayed Recall: a) 9-11 for subjects with $16+$ years of education, b) 5-9 for subjects with 8-15 years of education, or c) 3-6 for subjects with 0-7 years of education.

AD patients required an MMSE of 20-26 and a CDR of 0.5-1.0. AD patients must have achieved one of the following scores on Logical Memory Delayed Recall: a) $\leq 8$ for subjects with $16+$ years of education, b) $\leq 4$ for subjects with $8-15$ years of education, or c) $\leq 2$ for subjects with $0-7$ years of education.

Subjects were excluded at screening if they presented with major depression, bipolar disorder, a history of schizophrenia, or a history of alcohol abuse within the past two years. Due to the use of MRI in the ADNI protocol, subjects with partially metallic objects in their bodies (e.g. pacemakers) were excluded. Subjects with significant neurological disorders (excepting AD) were also excluded, including Parkinson's disease, multiple sclerosis, or significant head trauma.

\section{Measures}

\section{Everyday Cognition (ECog):}

The ECog is a 39-item questionnaire designed for informants to describe cognitive and functional decline. It has been adapted for patient self-ratings in these areas. Memory is represented as a single construct ("Everyday Memory”), made up of 8 questions. In contrast, Executive Functioning is represented in three parts: "Everyday 
Planning" (5 questions), "Everyday Organization" (6 questions), and "Everyday Divided Attention" (4 questions). ECog Global ratings (composed of all 39 questions) are significantly correlated with age $(r=0.19, p<0.05)$ and education $(r=-0.16, p<0.05)$, but neither ECog memory ratings or the three ECog executive function ratings are correlated with age or education. The ECog has been shown as reliable over a period of several months, as well as high in convergent validity with the CDR (Farias et al., 2008, 2013).

ECog ratings are made on a 1-4 scale, with the following options:

1: better or no change compared to 10 years earlier

2: questionable/occasionally worse

3: consistently a little worse

4: consistently much worse

Higher ratings on the ECog indicate worse daily function. In addition, the patient or informant had the option to answer any question with "I don't know." Given the structured nature of ADNI data collection, this response was uncommon $(<1 \%$ responded "I don't know" for most questions), but was coded as 0 when it appeared. As a measure of awareness, the ECog was operationalized as a discrepancy score between subject and informant rating. The discrepancy calculation took the form of Informant Score - Subject Score, which yielded larger positive numbers when the informant rated higher everyday impairment than the subject. 


\section{Geriatric Depression Scale - Short Form (GDS):}

Due to previous research indicating a correlation between increased depression and increased memory complaints (Minett, Da Silva, Ortiz, \& Bertolucci, 2008; Smith, Petersen, Ivnik, Malec, \& Tangalos, 1996), depression was included as a covariate. The GDS is a brief self-report measure designed to detect depression among older adults. It has been shown to be effective in the context of physical illness as well as mild and moderate stages of dementia (Sheikh \& Yesavage, 1986). The short form of the GDS has been shown to be a reliable and valid screening for depression (Almeida \& Almeida, 1999). The GDS was administered as a direct interview with the subject alone. The questionnaire contains 15 Yes or No questions and asks the subject to reflect on how they have felt over the past week. Scores between 0-5 indicate little to no evidence of depression. Scores of 6-15 suggest evidence of depression, with higher numbers indicating more severe depression.

Trail Making Test Parts A and B (Trails A and B):

Trails A and B are the two components of a measure of executive function and processing speed. Both components require attention, visuomotor skill, and perceptualscanning under time pressure. Part B also requires substantial cognitive flexibility (Reitan, 1958). More time spent to complete both Part A (150-second maximum) and Part B (300-second maximum) indicates greater impairment. Part A includes 25 circles numbered 1-25, while Part B includes 13 circles containing numbers (1-13) and 12 circles containing letters (A-L). In Part A, the subject must connect the circles in ascending numerical order, while in Part B, they must alternate connecting circles in ascending numerical and alphabetical order (1,A, 2, B, 3, C, etc.). Subjects are immediately 
corrected by the evaluator if they make an error, and urged to think further on what comes next in the pattern, e.g. "You were at number two. What is the next letter?" Although Trails B is the only one of these two scores directly measured in this study, subjects must complete Trails A immediately prior to Trails B for a standardized administration. Trails A and B have been shown to have both high test-retest reliability and high validity in detecting intact working memory, visuospatial ability, and taskshifting ability (Giovagnoli et al., 1996; Sánchez-Cubillo et al., 2009).

\section{Clock Drawing Test:}

The Clock Drawing Test measures constructional ability and skill at following a sequence of verbal directions. It was included as a measure of executive functioning skill, where it has been shown to be both valid as a dementia screening and high in test-retest reliability (Brodaty \& Moore, 1997; Ismail, Rajji, \& Shulman, 2010; Schmidtke \& Olbrich, 2007; Shulman, 2000). Subjects are provided a blank sheet of paper and instructed to "Draw the face of a clock showing the numbers and two hands set to ten after eleven" (Goodglass \& Kaplan, 1983). It has been shown to distinguish between healthy cognition and AD among older adults (Cahn-Weiner et al., 1996). In the ADNI protocol, scores range from $0-5$, with 1 point awarded for each of the following criteria: 1) an approximately circular face, 2) symmetrical number placement, 3) correct number order, 4) the presence of two (and only two) hands, and 5) the shorter hand must point to eleven and the longer hand must point to two. Lower scores indicates greater impairment. Alzheimer's Disease Assessment Scale-Cognitive (ADAS-Cog):

The ADAS-Cog was included as a measure of general cognition, with a focus on identifying cognitive and non-cognitive behavioral dysfunction associated with $\mathrm{AD}$ 
(Rosen, Mohs, \& Davis, 1984). The ADAS-Cog has been shown to be both valid and reliable in detecting cognitive change, albeit with evidence of ceiling effects on some subtests (Cano et al., 2010; Monllau et al., 2007; Weyer, Erzigkeit, Kanowski, Ihl, \& Hadler, 1997). It includes elements of learning, memory, language production, language comprehension, constructional praxis, ideational praxis, and orientation. This study used the inclusive scoring ( 85 point) method, which incorporates the following subtests: Word Recall (Immediate and Delayed), Commands/Instructions, Constructional Praxis, Naming, Ideational Praxis, Orientation, Word Recognition, Remembering Test Instructions and Comprehension, Word Finding Difficulty and Spoken Language Ability, and Number Cancellation. A higher total (i.e. more errors) indicates greater impairment. $\underline{\text { Rey Auditory Verbal Learning Test (RAVLT): }}$

The RAVLT was included as a test of learning and episodic memory (Rey, 1964). It contains five word list learning trials, an immediate recall trial following an interference word list, and recall and recognition trials following a 30-minute delay. The recognition trial is presented as a paper sheet containing a combination of words from the original list and novel words: The subject must circle only the words that were part of the original list. Lower scores (i.e. lower number of words recalled and higher numbers of false positives on recognition) indicate greater impairment. The RAVLT has shown high validity in discriminating between dementia syndromes and moderate test-retest reliability over a one-year period (Macartney-Filgate, 1988; Ricci, Graef, Blundo, \& Miller, 2012; Tierney, Nores, Snow, \& Fisher, 1994). 


\section{Logical Memory Test Immediate and Delayed Recall (Logical Memory):}

Logical Memory is a modified version of the Logical Memory measure of the Wechsler Memory Scale - Revised (Wechsler, 1987) and a test of episodic memory. Subjects hear a single short story and are then asked to immediately recall as much of the story as possible to the examiner, with a maximum score of 25 points awarded for complete recall of the story content. Subjects are advised that they will be asked to recall the story later in the testing. After a 30-minute delay, subjects are again asked to provide as much of the story as they can recall, with a maximum score of 25 . Lower scores in both phases of Logical Memory are indicative of greater impairment. Although independent reliability studies on this edition of the Logical Memory test are scarce, both the immediate and delayed recall components have demonstrated high validity in distinguishing memory performance (Leonberger, Nicks, Larrabee, \& Goldfader, 1992).

\section{Procedures}

All subjects completed clinical and cognitive assessment at a baseline study visit and for additional cognitive testing at a 12-month follow-up visit. A subset of the sample $(n=247)$ also completed 24-month follow-up cognitive testing. The study design was non-random (both in selection and assignment) and did not involve treatment for cognitive decline or dementia. Additional details regarding screening, biological sampling, neuroimaging, and the sequence of follow-up visits are available at: http://www.adni-info.org/Scientists/ADNIStudyProcedures.aspx 


\section{Statistical analysis}

All analyses were conducted using SPSS version 21 and AMOS version 21.

To examine whether lower executive functioning awareness at a baseline visit would predict lower scores on measures of executive functioning and memory performance at follow-up, linear regression analyses were employed. Specifically, the relationship between baseline ECog discrepancy scores and follow-up performance scores were studied, with gender, age, years of education, and GDS ratings included as covariates. The dependent variables were 12-month and 24-month follow-up performance (continuous) on the following measures: Trails B, Clock Drawing, ADAS-Cog, RAVLT, and Logical Memory. ECog Executive Functioning (the combined ratings of Everyday Planning, Everyday Organization, and Everyday Divided Attention) was the covariate of interest.

To examine whether lower executive functioning awareness at a baseline visit would yield a greater rate of diagnostic conversion at follow-up, logistic regression analyses were conducted. They were used to examine diagnostic change either from Normal Cognition to MCI or MCI to AD, at both 12-month and 24-month follow-up (See Subjects section above for diagnostic inclusion criteria). They employed the same predictors as in the above linear regression aside from neuropsychological test performance.

Finally, to examine whether poorer memory performance at a baseline visit increased the predictive ability of executive functioning awareness (yielding lower cognitive performance at follow-up), path analysis was conducted. To observe memory performance as a moderator, these analyses employed a two-group model. Using their 
baseline visit performance on Logical Memory Delayed Recall, subjects were split (a priori) into memory Impaired and Normal groups. Using a portion of the ADNI inclusion criteria for a diagnosis of "Cognitively Normal," subjects were categorized as Normal if their scores on Logical Memory - Delayed Recall were a) $\geq 9$ for subjects with $16+$ years of education, $b) \geq 5$ for subjects with $8-15$ years of education, or $\mathrm{c}) \geq 3$ for subjects with 0-7 years of education. Baseline discrepancy score on ECog Executive Functioning was an exogenous, continuous variable. It was used to predict 12-month and 24-month follow-up performance on Trails B, RAVLT, and ADAS-Cog. Constraints were individually applied to paths from baseline executive functioning discrepancy scores to follow-up Trails B, ADAS-Cog, and RAVLT performance in order to test for significant differences between the Normal and Impaired memory groups. Additionally, each model was evaluated for goodness of fit.

Although there is no gold standard awareness method in the literature (Roberts et al., 2009), memory ratings are frequent and make a useful comparison to executive functioning ratings. Therefore, linear and logistic regression analyses were also conducted with memory discrepancy score as a covariate. These additional analyses took two forms: 1) memory discrepancy replaced executive functioning discrepancy as the covariate of interest, and 2) both memory and executive functioning discrepancy were included as covariates. 


\section{RESULTS}

\section{Sample demographics}

Figures 2 and 3 are histograms of executive functioning and memory discrepancy scores, respectively. The executive functioning distribution had a mean of -1.11 (9.98) and the memory distribution had a mean of $-1.61(6.49)$. Both maintained an approximately normal distribution curve.

Table 1 displays the means and standard deviations of demographic, self-report, and neuropsychological scores of the 583 normal and MCI (53.0\% male) subjects involved in Hypotheses One and Two (linear regression and logistic regression, respectfully). The average age at the baseline visit was $72.1(\mathrm{SD}=7.1)$. The average total years of education was $16.3(\mathrm{SD}=2.6)$, indicating the average level of education was equivalent to a bachelor's degree. As expected given baseline recruitment criteria for the ADNI study, baseline GDS ratings were minimal, with a mean of $1.4(\mathrm{SD}=1.5)$.

Overall, scores on neuropsychological measures remained stable over time (baseline to month 12 to month $24, \mathrm{n}=583$ to $\mathrm{n}=231$ ). (Note: The decrease in sample size between time points does not represent sample attrition, but rather subjects that have only been in the study for 12-23 months.) T-test comparisons between these two groups' demographics, baseline discrepancy scores, and baseline performance scores did not show any significant differences.

Table 1 also contains Pearson correlations between each variable and the baseline discrepancy scores for both executive functioning and Memory. Executive functioning 
discrepancy was correlated with Logical Memory and RAVLT across all three time points, and ADAS-Cog at Baseline and Month 12. However, it was not correlated with the two executive functioning measures (Clock Drawing and Trails B), except Month 24 Trails B. Memory discrepancy was correlated with every neuropsychology measure except Month 24 Clock Drawing. Overall, the correlations for memory discrepancy scores appear either comparable or superior to the correlations for executive functioning discrepancy scores. All significant correlations for both executive functioning and Memory were in the expected direction, i.e. lower executive functioning and memory awareness was correlated with lower scores on each measure among both. (The correlations for Trails B and ADAS-Cog appear positive because higher totals on these measures represent poorer performance.) Both executive functioning and Memory discrepancy scores were also highly correlated with the other forms of baseline ECog measurement (executive functioning and memory subject self-ratings and study partner ratings).

Finally, Table 1 shows that baseline memory and executive functioning discrepancy scores were highly and positively correlated $(r=0.652, p<0.001)$. As an example of convergence across memory and executive functioning performance, poorer RAVLT and poorer Trails B were highly correlated at both Month $12(r=-0.313, p<$ $0.001)$ and Month $24(r=-0.315, p<0.001)$. Greater executive functioning discrepancy correlated more highly with poorer RAVLT at Month $12(r=-0.156, p<0.001)$ than poorer Trails B at Month $12(r=0.032, p=0.437)$. In contrast, higher executive functioning discrepancy correlated similarly with poorer RAVLT at Month $24(r=-0.202$, $p=0.002)$ and poorer Trails B at Month $24(r=0.155, p=0.016)$. Greater Memory 
discrepancy correlated with poorer Trails B at both Month $12(r=0.173, p<0.001)$ and Month $24(r=0.146, p=0.024)$. Overall, these correlations demonstrate modest convergent validity and low discriminant validity for executive functioning discrepancy scores (in the context of memory discrepancy scores) as predictors of future cognitive decline.

Hypothesis One: Lower executive functioning awareness (as demonstrated via discrepancy score) at a baseline visit will predict lower scores on measures of executive functioning and memory performance at 12-month and 24-month follow-up.

Table 2 details scores on executive functioning discrepancy and other baseline ECog ratings as predictors of poorer scores on Logical Memory (Delayed Recall) at Month 12 and Month 24, while controlling for the effects of age, gender, education, baseline GDS, and baseline Logical Memory score. The model as a whole explained approximately $57 \%$ of the variance among Month 12 Logical Memory scores $\left(R^{2}=0.57\right)$. The regression coefficient for executive functioning discrepancy $(\beta=-0.077, t=-2.768, p$ $=0.006)$ indicated that poorer executive functioning awareness predicted poorer Logical Memory scores at follow-up. Additionally, the regression coefficients for gender $(\beta=$ $0.071, t=2.514, p=0.012)$, age $(\beta=-0.104, t=-3.686, \quad p<0.001)$, and education $(\beta$ $=0.058, t=2.016, p=0.044)$ indicated that being older, male, and having fewer years of education predicted poorer Logical Memory scores at follow-up. Executive functioning discrepancy was not a significant predictor for change in Logical Memory scores at Month 24. Table 2 also details a significant Month 12 prediction for study partner rating $(\beta=-0.076, t=-2.536, p=0.011)$. In this instance, gender, age, and education remained 
significant predictors in the same directions as in the original executive functioning discrepancy model. The model accounted for a similar level of variance $\left(R^{2}\right.$ of 0.57$)$.

Table 3 details scores on executive functioning discrepancy and other baseline ECog ratings as predictors of poorer scores on Trails B at Month 12 and Month 24, while controlling for the effects of age, gender, education, baseline GDS, and baseline Trails B score. The Month 12 model was not significant. However, the Month 24 model was significant and explained approximately $27 \%$ of the variance in Month 24 scores. The regression coefficient for executive functioning discrepancy $(\beta=0.126 . t=2.185, p=$ 0.030) indicated that poorer executive functioning awareness predicted poorer Trails B scores. Additionally, the regression coefficients for age $(\beta=0.131, t=2.129, p=0.034)$ and baseline GDS $(\beta=0.117, t=1.997, p=0.047)$ indicated that being older and having more symptoms of depression predicted poorer Trails B scores. Table 3 also details a trend level Month 24 ECog prediction for subject rating $(\beta=0.106, \mathrm{t}=1.774, p=0.077)$ and a significant prediction for study partner rating $(\beta=0.228, \mathrm{t}=3.973, p<0.001)$. In each instance, increased age was a significant predictor of poorer Trails B score, but there were no additional predictions from baseline GDS. Each model accounted for similar, acceptable levels of variance $\left(R^{2}\right.$ of $\left.0.27-0.30\right)$.

Table 4 details scores on executive functioning discrepancy and other baseline ECog ratings as predictors of poorer scores on RAVLT (Delayed Recall) at Month 12 and Month 24, while controlling for the effects of age, gender, education, baseline GDS, and baseline RAVLT score. The model as a whole explained approximately $61 \%$ of the variance in Month 12 scores. The regression coefficient for executive functioning discrepancy $(\beta=-0.060, t=-2.244, p=0.025)$ indicated that poorer executive functioning 
awareness predicted poorer RAVLT scores. Additionally, the regression coefficients for age $(\beta=-0.105, t=-3.803, p<0.001)$, education $(\beta=0.048, t=2.987, p=0.003)$, and baseline GDS ( $\beta=0.086, t=-3.385, p=0.001)$ indicated that being older, having fewer years of education, and having more symptoms of depression predicted poorer RAVLT scores. Executive functioning discrepancy was not a significant predictor for change in RAVLT scores at Month 24. Table 4 also details a significant Month 12 ECog prediction for study partner rating $(\beta=-0.087, t=-3.120, p=0.002)$. In this instance, age, education, and baseline GDS were significant predictors in the same directions as in the executive functioning discrepancy model. The model accounted for a similar, high level of variance $\left(R^{2}\right.$ of 0.61$)$.

Table 5 details scores on executive functioning discrepancy and other baseline ECog ratings as predictors of poorer scores on ADAS-Cog (13-item Inclusive Scoring) at Month 12 and Month 24, while controlling for the effects of age, gender, education, baseline GDS, and baseline ADAS-Cog score. The Month 12 model as a whole explained approximately $65 \%$ of the variance in Month 24 ADAS-Cog scores. The regression coefficient for executive functioning discrepancy $(\beta=0.076, t=3.018, p=0.003)$ indicated that poorer executive functioning awareness predicted poorer ADAS-Cog scores at Month 12. Similarly, the Month 24 model as a whole explained approximately $64 \%$ of the variance in Month 24 ADAS-Cog scores. The regression coefficient for executive functioning discrepancy $(\beta=0.074, t=1.806, p=0.072)$ indicated that poorer executive functioning awareness predicted (at trend-level) poorer ADAS-Cog scores at Month 24. None of the regression coefficients aside from baseline ADAS-Cog score predicted change in follow-up ADAS-Cog score in either model. Table 5 also details 
significant predictions for ECog study partner ratings at both Month $12(\beta=0.091, t=$ 3.443, $p=0.001)$ and Month $24(\beta=0.087, t=2.046, p=0.042)$. In both instances, only baseline ADAS-Cog and the listed ECog score were significant predictors. Each model accounted for similar, high levels of variance ( $R^{2}$ of 0.65 in both models).

Table 6 details scores on executive functioning discrepancy and other baseline ECog ratings as predictors of poorer scores on Clock Drawing at Month 12 and Month 24, while controlling for the effects of age, gender, education, baseline GDS, and baseline Clock Drawing score. Executive functioning discrepancy was not a significant predictor for change in Clock Drawing scores at either time point.

Hypothesis Two: Lower executive functioning awareness (as demonstrated via discrepancy score) at a baseline visit will yield a greater rate of diagnostic conversion from normal cognition toward MCI and AD at 12-month and 24-month follow-up.

Table 7 details scores on executive functioning discrepancy and other baseline ECog ratings as predictors of diagnostic conversion (normal to $\mathrm{MCI}$, normal to $\mathrm{AD}$, or MCI to AD) from the baseline visit to Month 12, while controlling for the effects of age, gender, education, and baseline GDS. The model as a whole explained approximately $2 \%$ of the variance in diagnostic change $\left(R^{2}=0.02\right)$. Among the 583 subjects included in the Month 12 analyses, 43 converted to a more severe diagnosis. Among the 231 subjects included in the Month 24 analyses, 32 converted. Employing the odds ratio as a measure of effect size (Field, 2009), every point increase on executive functioning discrepancy was associated with a $1.05 \mathrm{x}$ increased likelihood of converting diagnosis $(\mathrm{OR}=1.05$, 95\% CI [1.02-1.08], $p=0.003)$. More symptoms of depression on the baseline GDS were 
also a significant predictor of diagnostic conversion $(\mathrm{OR}=1.23$, CI [1.01-1.51], $p=$ 0.040). However, gender, age, and education were not significant. Executive functioning discrepancy was not a significant predictor for diagnostic conversion at Month 24 .

Table 7 also details significant ECog predictions for Month 12 subject ratings $(\mathrm{OR}=1.04,95 \% \mathrm{CI}[1.00-1.08], p=0.030)$ and Month 12 study partner ratings $(\mathrm{OR}=$ $1.07,95 \% \mathrm{CI}[1.04-1.10], p<0.001)$, as well as a trend-level prediction for Month 24 study partner ratings $(\mathrm{OR}=1.04,95 \% \mathrm{CI}[1.00-1.07], p=0.055)$. In each instance, gender, age, education, and GDS were not significant predictors. The odds ratios of the models indicated a 1.04-1.07x increased likelihood of converting diagnosis, and the models accounted for $2-4 \%$ of the variance $\left(R^{2}\right.$ of $\left.0.02-0.04\right)$.

Hypothesis Three: Poorer memory performance at the baseline visit will increase the predictive ability of executive functioning awareness, yielding lower scores on both measures of executive functioning and memory performance at 12-month and 24-month follow-up.

To examine whether poorer memory performance at a baseline visit would increase the predictive ability of executive functioning awareness (yielding lower cognitive performance at follow-up), path analysis was used. These analyses employed a two-group model in order to observe memory performance as a moderator. The path analysis model contained the baseline ECog executive functioning discrepancy score as an exogenous variable. It was used to predict both baseline and either Month 12 or Month 24 follow-up scores on Trails B, ADAS-Cog, and RAVLT as endogenous variables. Clock Drawing was excluded as a variable due to the lack of significant relationships in 
linear regression. Logical Memory was excluded as a variable because the score was used to create the a priori moderator.

Table 8 contained the demographics for the slightly larger sample used in this analysis $(n=661)$. This is larger than the previous sample because subjects with an AD diagnosis at baseline are now included. These AD subjects were excluded from the previous regression analyses due to their focus on predicting decline from normal or mildly impaired cognition. In the path analyses of Hypothesis Three, the goal was to observe memory moderating decline in executive functioning awareness prediction at any stage of impairment. The Month 12 model compared two groups formed a priori: Normal memory $(n=444)$ and Impaired memory $(n=217)$, for a total of 661 individuals. This model demonstrated excellent fit, $(\chi 2(4)=2.758, p=0.599 ; \chi 2 / \mathrm{df}=0.690 ; \mathrm{CFI}=1.00$; RMSEA $<0.01)$. There were no significant differences between the Normal memory and Impaired memory groups when constraints were individually applied to paths from baseline executive functioning discrepancy scores to follow-up Trails B, ADAS-Cog, and RAVLT performance (see Table 9). In the Normal memory group, poorer baseline executive functioning awareness predicted poorer ADAS-Cog and RAVLT scores at Month 12. It was also associated with poorer baseline RAVLT scores (see Figure 4). In the Impaired memory group, poorer baseline executive functioning awareness showed a trend-level prediction for poorer ADAS-Cog scores at Month 12. It was also associated with poorer baseline scores among all three measures (see Figure 5). Constraining these paths to equality across the two groups did not significantly change model fit, which suggests the paths are not significantly different between the two groups. 
Next, the Month 24 model compared Normal memory $(\mathrm{n}=202)$ and Impaired memory $(n=45)$, for a total of 247 individuals. The Month 24 model demonstrated good fit, $(\chi 2(2)=9.902, p=0.042 ; \chi 2 / \mathrm{df}=2.476 ; \mathrm{CFI}=0.990 ; \mathrm{RMSEA}=0.08)$ Once again, there were no significant differences between the Normal memory and Impaired memory groups when constraints were individually applied to the paths from baseline executive functioning discrepancy to follow-up Trails B, ADAS-Cog, and RAVLT (see Table 9). In the Normal memory group, baseline executive functioning discrepancy showed a trend level prediction for poorer Month 24 Trails B scores, as well as a significant association with poorer baseline RAVLT scores (see Figure 6). In the Impaired memory model, baseline executive functioning discrepancy showed no significant predictions for Month 24 measures or associations with baseline measures (see Figure 7).

Uniqueness of executive functioning awareness in the context of memory awareness Memory prediction alone. Memory awareness discrepancy scores have shown moderate associations with cognitive decline in previous awareness studies (Clare, 2004a), and memory performance is critical to diagnosing both MCI and AD (Albert et al., 2011; Welsh-Bohmer \& Warren, 2006). Therefore, these scores were included in follow-up analyses in order to compare them to this study's more novel executive functioning awareness discrepancy scores. Poorer memory discrepancy scores predicted poorer follow-up performance on Logical Memory at Month $12(\beta=-0.089, t=-3.177, p$ $=0.002)$ and at Month $24(\beta=-0.103, t=-2.012, p=0.045$; see Table 10). There were no significant predictions from memory discrepancy scores on Trails B at Month 12 or Month 24 (see Table 11). Poorer memory discrepancy scores predicted poorer follow-up 
performance on RAVLT at Month $12(\beta=-0.067, t=-2.495, p=0.013$; see Table 12) but were not significant at Month 24. Poorer memory discrepancy scores predicted poorer follow-up performance on ADAS-Cog at Month $12(\beta=-0.096, t=3.770, p<0.001)$ and at Month $24(\beta=0.110, t=2.678, p=0.008$; see Table 13). Finally, poorer memory discrepancy scores predicted poorer follow-up performance on Clock Drawing at Month $12(\beta=-0.081, t=-2.178, p=0.030$; see Table 14) but were not significant at Month 24 . In logistic regression analyses, poorer memory awareness predicted diagnostic conversion at Month $12(\beta=1.105$, Wald $=16.854, p<0.001)$ and at Month $24(\beta=$ 1.070, Wald $=4.863, p=0.027$; see Table 15).

Linear regression combination. In order to examine the uniqueness of executive functioning awareness in the context of memory awareness, additional models were constructed. These re-estimated models examined executive functioning and memory discrepancy scores simultaneously. Each of the executive functioning discrepancy analyses described under Hypothesis One was re-estimated to include memory discrepancy as a covariate (see Table 16). Both discrepancy scores were highly correlated with one another $(r=0.652)$. In the Month 12 Logical Memory model, memory discrepancy was a trend-level predictor $(\beta=-0.670, t=-1.825, p=0.069)$, but executive functioning discrepancy prediction was not significant. Gender, age, and education were significant or trend-level predictors in the same directions as in the executive functioning discrepancy-alone model. This model also accounted for similar variance in cognitive performance to the original executive functioning awareness-alone prediction model $\left(R^{2}\right.$ of 0.58). In the Month 24 Trails B model, neither executive functioning or memory discrepancy were significant predictors. As in the executive functioning discrepancy- 
alone model, increased age and elevated baseline GDS predicted poorer Trails B scores. This model also accounted for similar variance in cognitive performance ( $R^{2}$ of 0.27$)$. In the Month 12 RAVLT model, neither executive functioning or memory discrepancy were significant predictors. Age, education, and baseline GDS were significant predictors in the same directions as in the executive functioning discrepancy-alone model. This model also accounted for similar variance in cognitive performance $\left(R^{2}\right.$ of 0.61$)$. In the Month 12 ADAS-Cog model, memory discrepancy was a significant predictor $(\beta=0.079, t=$ 2.372, $p=0.018$ ), but executive functioning discrepancy was not significant. In the Month 24 ADAS-Cog model, memory discrepancy was a trend-level predictor $(\beta=$ $0.105, t=1.964, p=0.051)$, but executive functioning discrepancy was not significant. In both Month 12 and 24 ADAS-Cog models, age, education, gender, and depression were not significant. Both models accounted for similar levels of variance in cognitive performance $\left(R^{2}\right.$ of $\left.0.64-0.65\right)$.

Logistic regression combination. Similar to the linear regression models above, the logistic models of executive functioning discrepancy prediction described under Hypothesis Two were re-estimated to include memory discrepancy as a covariate (see Table 16). In the Month 12 model, memory discrepancy was a significant predictor (OR $=1.101,95 \%$ CI [1.03-1.18], $p=0.004)$, but executive functioning discrepancy was not significant. There was also a trend for GDS prediction for memory discrepancy $(\mathrm{OR}=$ $1.22,95 \%$ CI $[0.99-1.49] p=0.055)$. Similar to the original model, this version accounted for minimal variance in diagnostic change $\left(R^{2}\right.$ of 0.04$)$. Neither executive functioning or memory discrepancy was predictive at Month 24. 


\section{DISCUSSION}

Previous research has shown many cross-sectional relationships and several longitudinal predictions from memory awareness to cognitive decline (Clare, 2010; Ecklund-Johnson \& Torres, 2005; Reid \& Maclullich, 2006; Retz-Junginger, Supprian, Retz, Rösler, \& Traue, 2005). Studies have also shown that both executive functioning performance and global awareness decline over time (Amanzio et al., 2013; Mathalon et al., 2003; Ott et al., 1996; Suchy et al., 2011; Van Wielingen et al., 2004). However, there has not yet been evidence of executive functioning awareness predicting longitudinal decline in memory or executive functioning performance, or of executive functioning awareness predicting change in diagnostic status. The following subsections will address the predictive ability of executive functioning awareness across this study's three research questions.

Research Question One: Can older adults' executive functioning awareness serve as a predictor of future cognitive decline?

Hypothesis One stated that lower executive functioning awareness (as demonstrated via discrepancy score) at a baseline visit would predict lower scores on measures of executive functioning and memory performance at 12-month and 24-month follow-up. This hypothesis was supported in a majority of measures. Poorer executive functioning awareness discrepancy scores (study partner rating - subject rating) predicted 
lower scores (i.e. change) on Month 12 Logical Memory, RAVLT, and ADAS-Cog, as well as Month 24 Trails B and (trend-level) ADAS-Cog. This relationship was not accounted for by gender, age, education, baseline depression self-report (GDS), or baseline scores on the respective measures. These findings provide evidence that executive awareness may predict longitudinal decline for both memory and executive functioning performance.

A large number of negative discrepancy scores were also observed, wherein the subject rated their abilities less positively than their study partner. This resulted in an approximately normal distribution of scores. All of the results in this section arose from linear prediction, suggesting that more negative discrepancy scores are associated with less decline on testing. However, this relationship is somewhat misleading. Significant rating discrepancy in any direction (significant overestimation or underestimation of their abilities) may be considered poor awareness. For example, a hypothetical patient with some memory problems may believe these problems have both greater breadth and depth than objective testing suggests. He may have a general understanding that his memory is declining, but lack specific details. He should therefore be considered to have poor awareness, rather than be considered worried but (relatively) cognitively intact (Clare et al., 2013). Despite this finding, all significant correlations and predictions involving executive functioning or memory discrepancy scores were in the expected direction. This suggests that if the negative discrepancy scores were removed or replaced with a score of zero (no discrepancy), many findings would likely show higher significance.

A previous study found that variability in self-awareness of performance in independent activities of daily living (IADLs) is present even among cognitively healthy 
older adults (Suchy et al., 2011). The ECog was designed in part as an update to the assessment of IADLs, one that would separate self-report into domains like memory and executive functioning (Farias et al., 2008). Furthermore, these findings complement Antoine, Nandrino, and Billiet's (2013) cross-sectional study on awareness and cognitive performance. Their study found that, unlike other measured cognitive domains, poor memory awareness was correlated with poor executive functioning performance. The present study reveals a different relationship, with poor executive function awareness predicting poor memory performance. Therefore, poor executive functioning awareness appears linked to both memory measures like Logical Memory and RAVLT and executive functioning measures like Trails B. Overall, the present study's findings suggest this longitudinal prediction method may provide earlier warnings of future decline than the more general questions of IADL functioning. The patient or their informant can endorse executive functioning difficulty alone, rather than difficulty with a complex task requiring multiple cognitive domains. Performance on tasks that require multiple domains is more difficult to interpret, as it is less clear how much impairment is due to one domain (e.g. memory) versus another (e.g. visuospatial ability).

The Clock Drawing Test measures executive functioning and has been demonstrated as a valid dementia screening and high in test-retest reliability (Brodaty \& Moore, 1997; Ismail et al., 2010; Schmidtke \& Olbrich, 2007; Shulman, 2000). However, there were no significant relationships between executive functioning discrepancy score and Clock Drawing score. This may be due in part to ceiling effects. Average scores were very high on this measure, both among the full study sample and the sample with $\mathrm{AD}$ patients removed (see Tables 1 and 8). Despite the robust connections between this 
measure and executive functioning in previous literature, it may be an ineffective outcome measure for the milder impairment stages explored in this study (MCI and mild $\mathrm{AD})$.

Greater executive functioning discrepancy scores at baseline was associated with poorer Logical Memory and RAVLT exclusively at Month 12 . Neuropsychology scores remained relatively stable across all three time points (see Table 1), thus it is somewhat surprising to see inconsistencies between time points in regression analyses, as no cohort differences were observed between the two time points ( $\mathrm{n}=583$ at Month 12 to $\mathrm{n}=231$ at Month 24). Additionally, reliability studies on Logical Memory are scarce and the RAVLT has questionable test-retest reliability after a 12-month period (MacartneyFilgate, 1988; Ricci et al., 2012; Tierney et al., 1994). Together, these factors may have disrupted predictions. Additionally, Trails B was predictive for Month 24 but not Month 12. Overt symptoms of executive functioning dysfunction often emerge later into $\mathrm{AD}$ progression than memory symptoms. Other components of Trails B, such as visuomotor skill and processing speed, do not typically decline in MCI or early stages of AD (WelshBohmer \& Warren, 2006). There may be a delay between subtle executive functioning decline in daily activities and overt decline on testing.

Executive functioning discrepancy predicted ADAS-Cog scores at Month 12 and at trend-level at Month 24. Given the ADAS-Cog's focus on behavioral dysfunction (Rosen et al., 1984), it may have had a stronger relationship with impaired executive functioning awareness compared to the memory measures. For example, maladaptive behavior is sign of dysfunction within the Cognitive Comparator Mechanisms (CCMs, the systems which identify new self-relevant errors; Morris \& Mograbi, 2013). Compared 
to the other measures, ADAS-Cog also has more robust evidence for validity and reliability in detecting subtle cognitive change (Cano et al., 2010; Monllau et al., 2007; Weyer et al., 1997). Despite the study's highly educated sample (average of 16.3 years), ceiling effects did not appear to disrupt the relationship to executive functioning awareness. The ADAS-Cog also takes approximately 30-45 minutes to administer, in comparison with other study measures requiring 5-15 minutes, allowing a greater breadth of memory and executive function evaluation.

Memory comparisons. Memory discrepancy was shown to be an effective predictor in every case where executive functioning discrepancy is an effective predictor except for Trails B Month 24. Memory discrepancy's broad success at prediction is expected, as poorer memory awareness indicating cognitive decline is relatively common in the literature (Clare, Whitaker, \& Nelis, 2010; Mitchell, 2008). Furthermore, executive functioning discrepancy scores were no longer significant predictors when included simultaneously in predictions with memory discrepancy scores. Among Pearson correlations, modest convergent validity and low discriminant validity were observed for executive functioning discrepancy scores in the context of memory discrepancy scores. This may indicate that the executive functioning discrepancy scores have little unique variance after taking memory awareness into consideration.

The strong correlation between memory and executive functioning discrepancy scores may also have explained why memory lost predictive ability for both Logical Memory and RAVLT when included in the simultaneous model. These two tests exclusively measured memory, and thus it was expected that baseline memory discrepancy would be a significant predictor unless variance was shared with a second, 
similar predictor. Alternatively, it is possible that these measures are simply less sensitive to poor memory awareness than the other study measures. The high scores on these measures (as observed in Table 1) suggest that ceiling effects may have reduced sensitivity. Future research in a sample less likely to ceiling (e.g. lower education) would add valuable perspective.

Research Question Two: Can older adults' executive functioning awareness serve as a predictor of diagnostic change?

Hypothesis Two stated that lower executive functioning awareness (as demonstrated via discrepancy score) at a baseline visit would predict a greater rate of diagnostic conversion from normal cognition toward MCI and $\mathrm{AD}$ at both 12-month and 24-month follow-up. Poorer baseline executive functioning awareness discrepancy scores predicted diagnostic conversion at Month 12 but not Month 24. This relationship was not accounted for by gender, age, education, or baseline GDS.

Many studies have compared awareness to neuropsychological test performance, but few have compared awareness to change in diagnostic category. This may be partly due to the heterogeneity of methods used to create a diagnosis (performance on screening measures, clinician ratings, baseline self or informant report, etc.) Such studies also risk becoming outdated as diagnostic criteria may be revised over time (Albert et al., 2011; Clare, 2010). As such, there is very little context available for these results in the extant literature. In a longitudinal study using informant-subject discrepancy ratings (Galeone, Pappalardo, Chieffi, Iavarone, \& Carlomagno, 2011), both MCI and mild AD were demonstrated to show reduced awareness compared to normal controls. However, MCI 
and $\mathrm{AD}$ scores were not significantly different from each other. These findings concur with the present study by suggesting discrepancy scores can help make a binary distinction between no impairment and some impairment.

In contrast to the linear regressions in Hypothesis One, $R^{2}$ values were very small $(0.02-0.04)$ in all models. Their small size may be related to the small number of diagnostic conversions overall - 43 of $583(7.4 \%)$ at Month 12, and 32 of $231(13.9 \%)$ at Month 24. As the ADNI study is still ongoing, data may emerge in the future to better explain these findings, e.g. post-mortem analyses, and determine if there were any confounds in the study demographics.

Memory comparisons. Unlike executive functioning discrepancy, memory discrepancy was an effective predictor for both time points. Moreover, in a model where memory and executive functioning discrepancy scores were both covariates, memory discrepancy remained a predictor at Month 12 and a trend-level predictor at Month 24, while executive functioning was not significant at either time point. Models of memory discrepancy both alone and with executive functioning discrepancy also contained very small $R^{2}$ values $(0.02-0.07)$, possibly reflecting the small number of conversions overall.

Research Question Three: Does older adults' objective memory performance moderate the relationship between executive functioning awareness and future cognitive decline?

It was hypothesized that poorer memory performance at the baseline visit would increase the predictive ability of executive functioning awareness, yielding lower scores on both measures of executive functioning and memory performance at 12-month and 24month follow-up. However, poorer memory performance at baseline did not increase the 
predictive ability of executive functioning awareness at either Month 12 or Month 24. A Logical Memory score below a cutoff (as detailed in by the ADNI guidelines reported in Methods) was used to approximate patients having memory difficulties consistent with MCI and mild AD. Poor episodic memory is known to manifest in MCI patients who progress to AD within several years (Albert et al., 2011). However, poor episodic memory does not guarantee poor awareness, as AD patients may use familiarity to retain awareness even as memory performance declines. This phenomenon has been observed for memory awareness and broad metacognition (Gallo et al., 2012; Pannu \& Kaszniak, 2005; Souchay, 2007) but not specifically in executive functioning awareness (see discussion of the CAM model below).

\section{Modeling the findings}

In the path analysis model, it was expected that dysfunction in episodic memory would hinder the flow of information into the executive system. In the CAM, the Cognitive Comparator Mechanisms (CCMs, the systems which identify new self-relevant errors) would have more difficulty detecting and comparing new errors to the personal database when memory is impaired (Morris \& Mograbi, 2013). However, there were no significant differences between the subjects with and without episodic memory dysfunction. These findings raise questions over component interaction within the CAM. For example, do older adults experience memory problems typical of mnemonic anosognosia, e.g. dysfunction in episodic memory and the personal database, but mild enough that healthy CCMs could correct them? Can some components be so impaired that no amount of assistance from other healthy components will resolve incorrect 
information? Finally, if one component is impaired but can be corrected by another healthy component, does this change the form of the metacognitive output generated at the top of the hierarchy?

The lack of significant differences may relate to the bi-directional flow of information within CAM (see Figure 1). Lower-order components like episodic memory can both influence and be influenced by higher-order components like the CCMs (Morris \& Mograbi, 2013). As there is no single awareness center in the CAM, healthy components can warn the subject (depending on their nature, either implicitly or explicitly) that an error has occurred. For example, if the episodic memory component is impaired but the CCMs are relatively functional, the subject may still be able to detect recent failures in poor memory and produce accurate executive functioning awareness. The awareness conflict is thus resolved before the information reaches metacognitive output, and the subject's explicit statements on awareness, via the ECog, become similar to their study partner's ratings.

Regarding the linear regression analyses, the successful prediction of both memory and executive functioning objective scores is also consistent with the CAM. The CCMs appear impaired when a subject's executive functioning awareness ratings at baseline are substantially more positive or negative than their future executive functioning performance. Dysfunction in the CCMs suggests additional dysfunction in the lower order components of episodic, working, and autobiographical memory. CAM does not offer any hypothesis on which form of anosognosia is most likely to precede the other in AD-related decline (Morris \& Mograbi, 2013). If mnemonic anosognosia preceded executive anosognosia in this sample, this might explain the normal 
distributions observed among discrepancy scores. Patients with mnemonic anosognosia sense a cognitive problem immediately after making an error. However, they quickly forget the specific details necessary to describe the nature and extent of the problem, which could result in both over and underestimation of deficit. Mnemonic anosognosia also represents a decline in episodic memory. If mnemonic anosognosia occurred near baseline, this would partially explain how poor awareness predicted poor performance on memory measures like Logical Memory and RAVLT at Month 12, but not on an executive functioning measure like Trails B until Month 24.

\section{Implications}

This study contributes to the existing awareness literature for MCI and AD by demonstrating that executive functioning awareness, as operationalized through a subject/informant discrepancy score, is a predictor of future decline on neuropsychological measures. These measures included a combination of memory and executive functioning tests. In contrast to the typically cross-sectional awareness designs, it included longitudinal measurements over a two-year period. It did so in a manner similar though not identical to the more thoroughly explored construct of memory awareness, establishing some convergent validity.

The subject/informant discrepancy score method of gauging awareness has shown moderate effectiveness in previous research (Clare, 2004a; Green et al., 1993), and may become more predictive when the questionnaires used ask intuitive questions about a single cognitive domain (Farias et al., 2008; Schinka, 2010). The moderate success of the 
method in this study, within both executive functioning and memory-based prediction, contributes to its overall construct validity as a measure of cognitive awareness.

Previous awareness research focused heavily on self-reported memory performance. The literature indicated memory awareness had moderate utility for detecting decline, particularly from normal cognition to $\mathrm{AD}$ (Clare, 2010). Consistent with this literature, this study also demonstrated executive functioning awareness as predictive in many of the same domains as memory awareness, e.g. verbal memory, attention, conceptualization, etc. Given the pattern of both executive functioning and memory awareness losing predictive ability when included simultaneously as covariates, both predictors appear relevant to obtaining a diagnosis. Based on linear regression analyses, executive functioning awareness via discrepancy scores has moderate predictive utility as a measure of cognitive decline. Based on logistic regression analyses, it also showed modest predictive utility in detecting change in diagnostic status. However, based on Pearson correlations and regression analyses containing both executive functioning and memory discrepancy scores, executive awareness does not appear unique from memory awareness.

The study findings also have implications in clinical settings. They provide evidence that brief assessment of executive functioning awareness discrepancy can predict worsening symptoms up to two years later, allowing a larger window for study and intervention. Low awareness of executive functioning may reach a primary care provider before low awareness of memory or other known forms of cognitive decline. For example, in linear regression models, poor baseline executive functioning discrepancy predicted Month 24 decline in Trails B but memory discrepancy did not. Although some 
results suggest that mnemonic anosognosia may have preceded executive anosognosia in this sample (see discussion of the CAM model above), this study does not confirm that memory awareness deteriorates prior to executive function awareness or vice versa. Regardless of the order of symptom presentation, primary care providers and family members may benefit from additional education on the cognitive deficits that appear to follow poor executive functioning awareness.

\section{Limitations}

Some limitations should be considered when interpreting study results. First, several potentially useful demographics items were unable to be included in the study, such as ethnicity. Ethnicity has informed decline on several disorders of aging (Curry \& Jackson, 2003) and would have been a useful covariate in both linear and logistic regressions. Study partner relationship status was also unavailable for a majority of subjects. Given that this study relies heavily on accurate informant reports, it would have been useful to observe the frequency of each relationship category (child, friend, significant other, etc.). This could have influenced the results if reports varied by study partner relationships, e.g. children providing lower overall estimations of subject abilities compared to friends. Additionally, this study's sample may not accurately reflect the current generation of older adults. For example, the sample had an average of 16.3 years of education, indicating high intellectual achievement. Socioeconomic status (SES) was also absent from the dataset. SES, along with education, has previously predicted cognitive decline. Conversely, higher SES and education are associated with better physical health (Adler \& Newman, 2002) and greater cognitive reserve (a reduced 
susceptibility to cognitive decline due to more efficient and versatile neural networks; Rentz et al., 2010; Stern, 2002; Suchy et al., 2011). Some ADNI criteria, such as the exclusion of subjects with a history of schizophrenia, may have influenced the sample toward higher SES and education (Byrne, Agerbo, Eaton, \& Mortensen, 2004). To increase generalizability, it would have been useful to include SES as an additional covariate and observe how it might vary with years of education.

Second, despite including age as a covariate in the linear and logistic models, there may have been generational differences within the study sample. The study age range of 55-90 includes multiple age cohorts, and previous literature among older adult samples has shown that older cohorts consider mental healthcare less important (Currin, Hayslip, Schneider, \& Kooken, 1998) and rate their overall health more favorably (Idler, 1993) than younger cohorts. This might have influenced results in the present study, e.g. younger subjects may have been more likely to report cognitive decline than older subjects. Cohort effects would be most disruptive for the logistic analyses because ADNI excludes subjects from the "cognitively normal" category if they have any subjective memory complaints (excepting mild declines associated solely with age).

Third, in the transition from Month 12 to Month 24, the study sample within the linear and logistic regressions decreased from 583 to 231, while the sample within the path analyses decreased from 661 to 247 . This is due to many subjects not being enrolled long enough in the ADNI study to reach their Month 24 visit. However, no differences between groups were expected or observed. As this ADNI phase is ongoing, future studies may be able to compare samples that are closer in size (with disparity due solely to attrition). 
Despite several limitations in sample characteristics and size, this study controlled for many other variables known to influence cognitive decline: age, gender, education, and symptoms of depression. This is the first study to examine executive functioning awareness as a predictor of longitudinal decline for MCI and AD. It is also the first to make direct comparisons between executive function awareness and memory awareness predictors among these diagnostic groups.

\section{Future Research Directions}

Future studies should continue to use longitudinal data. Longitudinal studies involving awareness discrepancy scores, both within subjects or between subjects and informants, are scarce (Clare et al., 2010; Reid \& Maclullich, 2006). Existing study designs, such as those of Gallo et al. (2012) and Ansell \& Bucks (2006) could be expanded to include subject/informant discrepancy scores and longitudinal observation.

Additionally, there exists substantial heterogeneity within the MCI diagnosis, even considering the recently revised NIA-AA diagnostic criteria requiring cognitive complaints for a patient and/or informant (Albert et al., 2011). The present study combined normal cognition and MCI subjects. Future studies may wish to compare more forms of MCI over time. For example, new studies might include a sample of late-stage MCI patients, who are more likely to convert over 1-2 years than normal controls or early-stage MCI patients (Collie et al., 2002). Given that this study found evidence of longitudinal executive functioning prediction in MCI and mild AD subjects, other disorders may also show this effect. Unlike AD, disorders such as frontotemporal dementia and Lewy body dementia often present with executive dysfunction but normal 
memory in their early stages (Welsh-Bohmer \& Warren, 2006). It would be useful to compare each of these disorders to the population represented in the present study over a period of several years. Executive functioning awareness may prove an even stronger predictor of cognitive decline in these dementia groups.

A detailed assessment of subject personality at baseline would complement the interview and depression scale used in the present study. For example, older adults with high levels of neuroticism are more likely to have cognitive complaints (Reid \& Maclullich, 2006) and general health complaints (Moor, Zimprich, Schmitt, \& Kliegel, 2006). Anxiety is also positively correlated with increased cognitive complaints among older adults with mild AD (Derouesné et al., 1999). Future studies might include a comprehensive personality measure, such as the Minnesota Multiphasic Personality Inventory-2 Restructured Form (MMPI-2-RF; Ben-Porath \& Tellegen, 2011).

Distinct among the results in Research Question One, executive functioning discrepancy score successfully predicted poorer Trails B performances scores at Month 24, while memory discrepancy was not a significant predictor at Month 12 or Month 24 . Future studies may benefit from comparing predictions for Trails B performance to predictions for additional measures of executive functioning, particularly more highceiling tasks such as the Wisconsin Card Sorting Task (WCST). The WCST is a measure of executive functioning and examines a subject's ability to organize, plan, and integrate frequent feedback (Stuss \& Levine, 2002). The Stroop task also measures executive functioning via cognitive flexibility and selective attention, and has a high ceiling (Van der Elst, Van Boxtel, Van Breukelen, \& Jolles, 2006). Finally, the Neuropsychological Assessment Battery (NAB) Executive Functions Module covers a broad range of 
executive functioning abilities, including problem solving, mental flexibility, and conceptualization. It also includes a subtest on daily activities and safety precautions, which would complement the everyday activities that make up the ECog (Stern \& White, 2003).

More novel forms of discrepancy scores, such as asking subjects to rate their performance both before and after a task (Ansell \& Bucks, 2006; Gallo et al., 2012; Hannesdottir \& Morris, 2007), could also be included in future investigations. This style of measurement is best suited to explore implicit awareness, which is thought to endure longer than the explicit awareness investigated via subjective discrepancy scores (Morris \& Mograbi, 2013). Subject/informant discrepancy is not interchangeable with other discrepancy methods, such as within-subject pre/post-task performance rating described above. Each type may provide distinct and useful information about a subject's impairment (Clare et al., 2013). Simultaneous, longitudinal measurements of both explicit and implicit awareness could confirm the existing CAM hypothesis that explicit awareness typically fades prior to implicit awareness.

Additionally, tools such as PET and fMRI would also expand the study of executive awareness. There is increasing evidence for the default mode network stagnating as $\mathrm{AD}$ progresses, including reduced activity in frontal regions (Zamboni \& Wilcock, 2011). Previous studies have established a connection between a compromised frontal lobe and increased executive dysfunction (McGlynn \& Schacter, 1989; Pannu \& Kaszniak, 2005), as well as a connection between higher amyloid burden and increased subjective memory complaints (Amariglio et al., 2012). Future research should attempt to determine if changes in cerebral blood flow, amyloid deposition, or brain volume 
correlate with changes in executive awareness, and if these correlations also exist for memory awareness.

Finally, future studies should expand the construct validity of executive functioning awareness to predict decline. Executive functioning awareness has a very limited history of prediction in research, and should be investigated with many more awareness operationalizations and outcome measures. As the literature grows, more direct comparisons to memory awareness can be made, which could increase discriminant validity between memory and executive functioning discrepancy scores. It may also be useful to include more questionnaires to examine more skills associated with the frontal lobe. The Socio-Emotional Questionnaire (SEQ) forms a subject/informant discrepancy score based on a subject's awareness of their emotion recognition, empathy, social conformity, antisocial behavior, and sociability (Bramham, Morris, Hornak, Bullock, \& Polkey, 2009). Decline in these areas is associated with frontal dysfunction (WelshBohmer \& Warren, 2006), and further examination may show a relationship to executive functioning awareness. Finally, by using multiple questionnaires, future studies could also establish cut scores. For example, subjects could be placed into an impaired category if they had a large aggregate discrepancy score (either toward over or underestimating their abilities). Impaired vs. normal executive functioning awareness could then be used as a predictor in place of the continuous discrepancy scores in the present study.

Although this study has expanded the scope of awareness as a diagnostic tool, many questions remain. The authors of the CAM noted that anosognosia as a whole has a heterogeneous presentation and the main causal factors are currently unknown (Morris \& Mograbi, 2013). The time course of early deficits in awareness, i.e. the first symptoms of 
mnemonic and executive anosognosia, must be investigated further. Currently, changes in executive functioning awareness appear highly relevant to both forms of anosognosia.

Studying these changes may be crucial to obtaining earlier and more accurate diagnoses of $\mathrm{MCI}$ and $\mathrm{AD}$. 


\section{REFERENCES}

Adler, N. E., \& Newman, K. (2002). Socioeconomic disparities in health: Pathways and policies. Health Affairs, 21(2), 60-76.

Agnew, S. K., \& Morris, R. G. (1998). The heterogeneity of anosognosia for memory impairment in Alzheimer's disease: A review of the literature and a proposed model. Aging \& Mental Health, 2(1), 7-19.

Albert, M. S., DeKosky, S. T., Dickson, D., Dubois, B., Feldman, H. H., Fox, N. C., ... Phelps, C. H. (2011). The diagnosis of mild cognitive impairment due to Alzheimer's disease: Recommendations from the National Institute on AgingAlzheimer's Association workgroups on diagnostic guidelines for Alzheimer's disease. Alzheimer's \& Dementia: The Journal of the Alzheimer's Association, 7(3), 270-9.

Almeida, O. P., \& Almeida, S. a. (1999). Short versions of the geriatric depression scale: A study of their validity for the diagnosis of a major depressive episode according to ICD-10 and DSM-IV. International Journal of Geriatric Psychiatry, 14(10), 85865.

Amanzio, M., Vase, L., Leotta, D., Miceli, R., Palermo, S., \& Geminiani, G. (2013). Impaired awareness of deficits in Alzheimer's disease: The role of everyday executive dysfunction. Journal of the International Neuropsychological Society: JINS, 19(1), 63-72.

Amariglio, R. E., Becker, J. A., Carmasin, J. S., Wadsworth, L. P., Lorius, N., Sullivan, C., ... Rentz, D. M. (2012). Subjective cognitive complaints and amyloid burden in cognitively normal older individuals. Neuropsychologia, 50(12), 2880-6.

Ansell, E. L., \& Bucks, R. S. (2006). Mnemonic anosognosia in Alzheimer's disease: A test of Agnew and Morris (1998). Neuropsychologia, 44(7), 1095-102.

Antoine, C., Antoine, P., Guermonprez, P., \& Frigard, B. (2004). [Awareness of deficits and anosognosia in Alzheimer's disease]. L'Encéphale, 30(6), 570-7.

Antoine, P., Nandrino, J.-L., \& Billiet, C. (2013). Awareness of deficits in Alzheimer's disease patients: Analysis of performance prediction discrepancies. Psychiatry and Clinical Neurosciences, 67(4), 237-44. 
Ben-Porath, Y. S., \& Tellegen, A. (2011). MMPI-2-RF (Minnesota Multiphasic Personality Inventory-2 Restructured Form): Manual for administration, scoring, and interpretation. Minneapolis, MN: University of Minnesota Press.Braak, H., \& Braak, E. (1991). Neuropathological stageing of Alzheimer-related changes. Acta Neuropathologica, 82(4), 239-59.

Bradford, A., Kunik, M. E., Schulz, P., Williams, S. P., \& Singh, H. (2009). Missed and delayed diagnosis of dementia in primary care: Prevalence and contributing factors. Alzheimer Disease and Associated Disorders, 23(4), 306-14.

Bramham, J., Morris, R. G., Hornak, J., Bullock, P., \& Polkey, C. E. (2009). Social and emotional functioning following bilateral and unilateral neurosurgical prefrontal cortex lesions. Journal of Neuropsychology, 3(Pt 1), 125-43.

Brodaty, H., \& Moore, C. (1997). The Clock Drawing Test for dementia of the Alzheimer's type: A comparison of three scoring methods in a memory disorders clinic. International Journal of Geriatric Psychiatry, 12(6), 619-627.

Buckner, R. L., Andrews-Hanna, J. R., \& Schacter, D. L. (2008). The brain's default network: Anatomy, function, and relevance to disease. Annals of the New York Academy of Sciences, 1124, 1-38.

Byrne, M., Agerbo, E., Eaton, W. W., \& Mortensen, P. B. (2004). Parental socioeconomic status and risk of first admission with schizophrenia- a Danish national register based study. Social Psychiatry and Psychiatric Epidemiology, 39(2), 87-96.

Cahn-Weiner, D. A., Salmon, D., Monsch, A. U., Butters, N., Wiederholt, W. C., CoreyBloom, J., \& Barrett-Connor, E. (1996). Screening for dementia of the alzheimer type in the community: The utility of the Clock Drawing Test. Archives of Clinical Neuropsychology: The Official Journal of the National Academy of Neuropsychologists, 11(6), 529-39.

Cano, S. J., Posner, H. B., Moline, M. L., Hurt, S. W., Swartz, J., Hsu, T., \& Hobart, J. C. (2010). The ADAS-cog in Alzheimer's disease clinical trials: Psychometric evaluation of the sum and its parts. Journal of Neurology, Neurosurgery, and Psychiatry, 81(12), 1363-8.

Chan, R. C. K., Shum, D., Toulopoulou, T., \& Chen, E. Y. H. (2008). Assessment of executive functions: Review of instruments and identification of critical issues. Archives of Clinical Neuropsychology: The Official Journal of the National Academy of Neuropsychologists, 23(2), 201-16.

Clare, L. (2004a). Awareness in early-stage Alzheimer's disease: A review of methods and evidence. The British Journal of Clinical Psychology / the British Psychological Society, 43(Pt 2), 177-96. 
Clare, L. (2004b). The construction of awareness in early-stage Alzheimer's disease: A review of concepts and models. The British Journal of Clinical Psychology / the British Psychological Society, 43(Pt 2), 155-75.

Clare, L. (2010). Awareness in people with severe dementia: Review and integration. Aging \& Mental Health, 14(1), 20-32.

Clare, L., Marková, I., Verhey, F., \& Kenny, G. (2005). Awareness in dementia: A review of assessment methods and measures. Aging \& Mental Health, 9(5), 394413.

Clare, L., Whitaker, C. J., \& Nelis, S. M. (2010). Appraisal of memory functioning and memory performance in healthy ageing and early-stage Alzheimer's disease. Neuropsychology, Development, and Cognition. Section B, Aging, Neuropsychology and Cognition, 17(4), 462-91.

Clare, L., Whitaker, C. J., Nelis, S. M., Martyr, A., Markova, I. S., Roth, I., ... Morris, R. G. (2011). Multidimensional assessment of awareness in early-stage dementia: A cluster analytic approach. Dementia and Geriatric Cognitive Disorders, 31(5), 31727.

Clare, L., Whitaker, C. J., Roberts, J. L., Nelis, S. M., Martyr, A., Marková, I. S., ... Morris, R. G. (2013). Memory awareness profiles differentiate mild cognitive impairment from early-stage dementia: Evidence from assessments of performance monitoring and evaluative judgement. Dementia and Geriatric Cognitive Disorders, 35(5-6), 266-79.

Collie, A., Maruff, P., \& Currie, J. (2002). Behavioral characterization of mild cognitive impairment. Journal of Clinical and Experimental Neuropsychology, 24(6), 720-33.

Cosentino, S., Metcalfe, J., Butterfield, B., \& Stern, Y. (2007). Objective metamemory testing captures awareness of deficit in Alzheimer's disease. Cortex; a Journal Devoted to the Study of the Nervous System and Behavior, 43(7), 1004-19.

Currin, J. B., Hayslip, B., Schneider, L. J., \& Kooken, R. A. (1998). Cohort differences in attitudes toward mental health services among older persons. Psychotherapy: Theory, Research, Practice, Training, 35(4), 506-518.

Curry, L., \& Jackson, J. (2003). Recruitment and retention of diverse ethnic and racial groups in health research: An evolving science. In L. Curry \& J. Jackson (Eds.), The science of inclusion: Recruiting and retaining racial and ethnic elders in health research (pp. 1-7). Washington, DC: Gerontological Society of America. 
Derouesné, C., Thibault, S., Lagha-Pierucci, S., Baudouin-Madec, V., Ancri, D., \& Lacomblez, L. (1999). Decreased awareness of cognitive deficits in patients with mild dementia of the Alzheimer type. International Journal of Geriatric Psychiatry, 14(12), 1019-30.

Ecklund-Johnson, E., \& Torres, I. (2005). Unawareness of deficits in Alzheimer's disease and other dementias: Operational definitions and empirical findings. Neuropsychology Review, 15(3), 147-66.

Farias, S. T., Mungas, D., Reed, B. R., Cahn-Weiner, D., Jagust, W., Baynes, K., \& Decarli, C. (2008). The measurement of everyday cognition (ECog): Scale development and psychometric properties. Neuropsychology, 22(4), 531-44.

Farias, S. T., Park, L. Q., Harvey, D. J., Simon, C., Reed, B. R., Carmichael, O., \& Mungas, D. (2013). Everyday cognition in older adults: Associations with neuropsychological performance and structural brain imaging. Journal of the International Neuropsychological Society: JINS, 19(4), 430-41.

Field, A. (2009). Discovering Statistics Using SPSS (3rd ed.). London: SAGE Publications Ltd.

Frank, L., Lenderking, W. R., Howard, K., \& Cantillon, M. (2011). Patient self-report for evaluating mild cognitive impairment and prodromal Alzheimer's disease. Alzheimer's Research \& Therapy, 3(6), 35.

Galeone, F., Pappalardo, S., Chieffi, S., Iavarone, A., \& Carlomagno, S. (2011). Anosognosia for memory deficit in amnestic mild cognitive impairment and Alzheimer's disease. International Journal of Geriatric Psychiatry, 26(7), 695-701.

Gallo, D. A., Chen, J. M., Wiseman, A. L., Schacter, D. L., \& Budson, A. E. (2007). Retrieval monitoring and anosognosia in Alzheimer's disease. Neuropsychology, 21(5), 559-68.

Gallo, D. A., Cramer, S. J., Wong, J. T., \& Bennett, D. a. (2012). Alzheimer's disease can spare local metacognition despite global anosognosia: Revisiting the confidenceaccuracy relationship in episodic memory. Neuropsychologia, 50(9), 2356-64.

Giovagnoli, A. R., Pesce, M., Mascheroni, S., Simoncelli, M., Laiacona, M., \& Capitani, E. (1996). Trail making test: Normative values from 287 normal adult controls. The Italian Journal of Neurological Sciences, 17(4), 305-309.

Goel, V., Grafman, J., Tajik, J., Gana, S., \& Danto, D. (1997). A study of the performance of patients with frontal lobe lesions in a financial planning task. Brain : A Journal of Neurology, 120 ( Pt 1), 1805-22. 
Goodglass, H., \& Kaplan, E. (1983). The assessment of aphasia and related disorders. Philadelphia: Lea \& Febiger.

Grafman, J., \& Litvan, I. (1999). Importance of deficits in executive functions. Lancet, 354(9194), 1921-3.

Green, J., Goldstein, F. C., Sirockman, B. E., \& Green, R. C. (1993). Variable Awareness of Deficits in Alzheimer's Disease. Cognitive and Behavioral Neurology, 6(3).

Hannesdottir, K., \& Morris, R. G. (2007). Primary and Secondary Anosognosia for Memory Impairment in Patients with Alzheimer's Disease. Cortex, 43(7), 10201030 .

Idler, E. L. (1993). Age differences in self-assessments of health: Age changes, cohort differences, or survivorship? Journal of Gerontology, 48(6), S289-S300.

Ismail, Z., Rajji, T. K., \& Shulman, K. I. (2010). Brief cognitive screening instruments: An update. International Journal of Geriatric Psychiatry, 25(2), 111-20.

Johnson, S. C., Ries, M. L., Hess, T. M., Carlsson, C. M., Gleason, C. E., Alexander, A. L., ... Sager, M. A. (2007). Effect of Alzheimer disease risk on brain function during self-appraisal in healthy middle-aged adults. Archives of General Psychiatry, 64(10), 1163-71.

Jurado, M. B., \& Rosselli, M. (2007). The elusive nature of executive functions: A review of our current understanding. Neuropsychology Review, 17(3), 213-33.

Klein, S. B., \& Lax, M. L. (2010). The unanticipated resilience of trait self-knowledge in the face of neural damage. Memory (Hove, England), 18(8), 918-48.

Leonberger, F. T., Nicks, S. D., Larrabee, G. J., \& Goldfader, P. R. (1992). Factor structure of the Wechsler Memory Scale--Revised within a comprehensive neuropsychological battery. Neuropsychology, 6(3), 239-249.

Lopez, O. L., Becker, J. T., Somsak, D., Dew, M. A., \& DeKosky, S. T. (1994). Awareness of cognitive deficits and anosognosia in probable Alzheimer's disease. European Neurology, 34(5), 277-82.

Macartney-Filgate, M. (1988). Intercorrelation of clinical tests of verbal memory. Archives of Clinical Neuropsychology, 3(2), 121-126.

Marková, I. S., \& Berrios, G. E. (2006). Approaches to the assessment of awareness: Conceptual issues. Neuropsychological Rehabilitation, 16(4), 439-55. 
Mathalon, D. H., Bennett, A., Askari, N., Gray, E. M., Rosenbloom, M. J., \& Ford, J. M. (2003). Response-monitoring dysfunction in aging and Alzheimer's disease: An event-related potential study. Neurobiology of Aging, 24(5), 675-85.

McGlynn, S. M., \& Schacter, D. L. (1989). Unawareness of deficits in neuropsychological syndromes. Journal of Clinical and Experimental Neuropsychology, 11(2), 143-205.

Mimura, M., \& Yano, M. (2006). Memory impairment and awareness of memory deficits in early-stage Alzheimer's disease. Reviews in the Neurosciences, 17(1-2), 253-66.

Minett, T. S. C., Da Silva, R. V., Ortiz, K. Z., \& Bertolucci, P. H. F. (2008). Subjective memory complaints in an elderly sample: A cross-sectional study. International Journal of Geriatric Psychiatry, 23(1), 49-54.

Mitchell, A. J. (2008). The clinical significance of subjective memory complaints in the diagnosis of mild cognitive impairment and dementia: A meta-analysis.

International Journal of Geriatric Psychiatry, 23(11), 1191-202.

Mograbi, D. C., Brown, R. G., \& Morris, R. G. (2009). Anosognosia in Alzheimer's disease--the petrified self. Consciousness and Cognition, 18(4), 989-1003.

Monllau, A., Pena-Casanova, J., Blesa, R., Aguilar, M., Bohm, P., Sol, J. M., \& Hernandez, G. (2007). [Diagnostic value and functional correlations of the ADASCog scale in Alzheimer's disease: Data on NORMACODEM project]. Neurología (Barcelona, Spain), 22(8), 493-501.

Moor, C., Zimprich, D., Schmitt, M., \& Kliegel, M. (2006). Personality, aging selfperceptions, and subjective health: A mediation model. International Journal of Aging \& Human Development, 63(3), 241-57.

Morris, R. G., \& Mograbi, D. C. (2013). Anosognosia, autobiographical memory and self knowledge in Alzheimer's disease. Cortex; A Journal Devoted to the Study of the Nervous System and Behavior, 49(6), 1553-65.

Ott, B. R., Lafleche, G., Whelihan, W. M., Buongiorno, G. W., Albert, M. S., \& Fogel, B. S. (1996). Impaired Awareness of Deficits in Alzheimer Disease. Alzheimer Disease \& Associated Disorders, 10(2), 68-76.

Pannu, J. K., \& Kaszniak, A. W. (2005). Metamemory experiments in neurological populations: A review. Neuropsychology Review, 15(3), 105-30.

Pia, L., \& Conway, P. (2008). Anosognosia and Alzheimer's Disease. Brain Impairment, $9(1), 22-27$. 
Purser, J. L., Fillenbaum, G. G., \& Wallace, R. B. (2006). Memory complaint is not necessary for diagnosis of mild cognitive impairment and does not predict 10-year trajectories of functional disability, word recall, or short portable mental status questionnaire limitations. Journal of the American Geriatrics Society, 54(2), 335-8.

Ready, R. E., Ott, B. R., Grace, J., \& Cahn-Weiner, D. A. (2003). Apathy and executive dysfunction in mild cognitive impairment and Alzheimer disease. The American Journal of Geriatric Psychiatry: Official Journal of the American Association for Geriatric Psychiatry, 11(2), 222-8.

Reid, L. M., \& Maclullich, A. M. J. (2006). Subjective memory complaints and cognitive impairment in older people. Dementia and Geriatric Cognitive Disorders, 22(5-6), 471-85.

Reitan, R. (1958). Validity of the Trail Making Test as an indicator of organic brain damage. Perceptual and Motor Skills, 8(3), 271-276.

Rentz, D. M., Locascio, J. J., Becker, J. A., Moran, E. K., Eng, E., Buckner, R. L., ... Johnson, K. A. (2010). Cognition, reserve, and amyloid deposition in normal aging. Annals of Neurology, 67(3), 353-64.

Retz-Junginger, P., Supprian, T., Retz, W., Rösler, M., \& Traue, H. C. (2005). [Awareness of memory deficits in patients with probable Alzheimer's disease]. Fortschritte Der Neurologie-Psychiatrie, 73(6), 327-32.

Rey, A. (1964). L'exarnen clinique en psychologie. Paris: Presses Universitaires de France.

Ricci, M., Graef, S., Blundo, C., \& Miller, L. A. (2012). Using the Rey Auditory Verbal Learning Test (RAVLT) to differentiate alzheimer's dementia and behavioural variant fronto-temporal dementia. The Clinical Neuropsychologist, 26(6), 926-41.

Roberts, J. L., Clare, L., \& Woods, R. T. (2009). Subjective memory complaints and awareness of memory functioning in mild cognitive impairment: A systematic review. Dementia and Geriatric Cognitive Disorders, 28(2), 95-109.

Rosen, W. G., Mohs, R. C., \& Davis, K. L. (1984). A new rating scale for Alzheimer's disease. The American Journal of Psychiatry, 141, 1356-1364.

Salmon, E., Ruby, P., Perani, D., Kalbe, E., Laureys, S., Adam, S., \& Collette, F. (2005). Two aspects of impaired consciousness in Alzheimer's disease. Progress in Brain Research, 150, 287-98. 
Sánchez-Cubillo, I., Periáñez, J. A., Adrover-Roig, D., Rodríguez-Sánchez, J. M., RíosLago, M., Tirapu, J., \& Barceló, F. (2009). Construct validity of the Trail Making Test: Role of task-switching, working memory, inhibition/interference control, and visuomotor abilities. Journal of the International Neuropsychological Society: JINS, 15(3), 438-50.

Saykin, A. J., Wishart, H. A., Rabin, L. A., Santulli, R. B., Flashman, L. A., West, J. D., ... Mamourian, A. C. (2006). Older adults with cognitive complaints show brain atrophy similar to that of amnestic MCI. Neurology, 67(5), 834-42.

Schinka, J. A. (2010). Use of informants to identify mild cognitive impairment in older adults. Current Psychiatry Reports, 12(1), 4-12.

Schmidtke, K., \& Olbrich, S. (2007). The Clock Reading Test: Validation of an instrument for the diagnosis of dementia and disorders of visuo-spatial cognition. International Psychogeriatrics / IPA, 19(2), 307-21.

Sheikh, J. I., \& Yesavage, J. A. (1986). Geriatric Depression Scale (GDS): Recent evidence and development of a shorter version. Clinical Gerontologist, 5, 165-173.

Shulman, K. I. (2000). Clock-drawing: Is it the ideal cognitive screening test? International Journal of Geriatric Psychiatry, 15(6), 548-61.

Smith, G. E., Petersen, R. C., Ivnik, R. J., Malec, J. F., \& Tangalos, E. G. (1996). Subjective memory complaints, psychological distress, and longitudinal change in objective memory performance. Psychology and Aging, 11(2), 272-279.

Souchay, C. (2007). Metamemory in Alzheimer's Disease. Cortex, 43(7), 987-1003.

Stern, R., \& White, T. (2003). Neuropsychological Assessment Battery: Administration, scoring, and interpretation manual. Lutz, FL: Psychological Assessment Resources.

Stern, Y. (2002). What is cognitive reserve? Theory and research application of the reserve concept. Journal of the International Neuropsychological Society : JINS, $8(3), 448-60$.

Stewart, G., McGeown, W. J., Shanks, M. F., \& Venneri, A. (2010). Anosognosia for memory impairment in Alzheimer's disease. Acta Neuropsychiatrica, 22(4), 180 187.

Stuss, D. T., \& Levine, B. (2002). Adult clinical neuropsychology: Lessons from studies of the frontal lobes. Annual Review of Psychology, 53, 401-33. 
Suchy, Y., Kraybill, M. L., \& Franchow, E. (2011). Instrumental activities of daily living among community-dwelling older adults: Discrepancies between self-report and performance are mediated by cognitive reserve. Journal of Clinical and Experimental Neuropsychology, 33(1), 92-100.

Tierney, M. C., Nores, A., Snow, W. G., \& Fisher, R. H. (1994). Use of the Rey Auditory Verbal Learning Test in differentiating normal aging from Alzheimer's and Parkinson's dementia. Psychological Assessment, 6(2), 129-134.

Van der Elst, W., Van Boxtel, M. P. J., Van Breukelen, G. J. P., \& Jolles, J. (2006). The Stroop color-word test: influence of age, sex, and education; And normative data for a large sample across the adult age range. Assessment, 13(1), 62-79.

Van Wielingen, L. E., Tuokko, H. a, Cramer, K., Mateer, C. a, \& Hultsch, D. F. (2004). Awareness of financial skills in dementia. Aging \& Mental Health, 8(4), 374-80.

Wechsler, D. (1987). Wechsler Memory Scale-Revised Manual. New York: The Psychological Corporation.

Weimer, D. L., \& Sager, M. a. (2009). Early identification and treatment of Alzheimer's disease: Social and fiscal outcomes. Alzheimer's \& Dementia: The Journal of the Alzheimer's Association, 5(3), 215-26.

Welsh-Bohmer, K. A., \& Warren, L. H. (2006). Neurodegenerative dementias. In D. K. Attix \& K. A. Welsh-Bohmer (Eds.), Geriatric neuropsychology: Assessment and intervention (pp. 56-88). New York: The Guilford Press.

Weyer, G., Erzigkeit, H., Kanowski, S., Ihl, R., \& Hadler, D. (1997). Alzheimers Disease Assessment Scale: Reliability and Validity in a Multicenter Clinical Trial. International Psychogeriatrics, 9(2), 123-138.

Zamboni, G., \& Wilcock, G. (2011). Lack of awareness of symptoms in people with dementia: The structural and functional basis. International Journal of Geriatric Psychiatry, 26(8), 783-92. 
Table 1

Study Sample Characteristics and Correlations with ECog Scales - Normal and MCI Subjects

Variable

Mean

SD

EF Disc

Pearson

EF Disc

Memory

Disc

Memory

Pearson

Disc

p-value

Age at Baseline

Education (years)

72.1

7.1

0.020

0.630

0.100*

0.015

GDS-Baseline

ECog EF Subject

$16.3 \quad 2.6$

$-0.031$

0.457

$-0.041$

0.322

$1.4 \quad 1.5$

$-0.096 *$

0.020

$-0.049$

0.234

ECog Memory Subject

22.8

$-0.507^{* * *}$

$<0.001$

$-0.251^{* * *}$

$<0.001$

ECog EF Study Partner

16.5

5.7

$-0.278^{* * *}$

$<0.001$

$-0.453^{* *}$

$<0.001$

ECog Memory Study Partner

23.0

$0.632^{* * *}$

$<0.001$

$\mathrm{0.483}^{\text {*** }}$

$<0.001$

ECog EF Discrepancy

$14.9 \quad 6.4$

$0.412^{* * *}$

$<0.001$

$0.609^{* * *}$

$<0.001$

ECog Memory Discrepancy

$-1.1 \quad 10.0$

$-1.6 \quad 6.5$

$0.652^{* *}$

$0.652^{* * *}$

$<0.001$

Logical Memory Baseline

Logical Memory Month 12

9.1

4.2

$-0.131^{\text {*** }}$

9.8

5.4

$-0.177^{* * *}$

0.002

$-0.183^{* *}$

$<0.001$

Logical Memory Month 24

10.2

$-0.176^{* * *}$

$<0.001$

$-0.238^{\text {*** }}$

$<0.001$

RAVLT Delayed Baseline

RAVLT Delayed Month 12

RAVLT Delayed Month 24

Clock Drawing Baseline

5.7

$-0.146^{* *}$

0.007

$-0.219^{* *}$

0.001

$5.5 \quad 4.6 \quad \mathbf{- 0 . 1 5 6}^{\text {** }}$

$<0.001$

$-0.231^{* *}$

$<0.001$

$5.1 \quad 4.5$

$-0.202^{* * *}$

$<0.001$

$-0.238^{* * *}$

$<0.001$

$\begin{array}{ll}4.5 & 0.8\end{array}$

$-0.063$

0.002

$-0.199^{* * *}$

0.002

$4.6 \quad 0.8$

0.131

$-0.128^{* *}$

0.002

Clock Drawing Month 12

Clock Drawing Month 24

4.6

$-0.062$

0.136

$-0.145^{* *}$

$<0.001$

Trails Baseline

Trails Month 12

97.2

0.7

$-0.022$

0.737

$-0.048$

0.461

0.035

0.399

$0.179 * *$

$<0.001$

100.7

0.032

0.437

$0.173^{\text {*** }}$

$<0.001$

$99.8 \quad 80.0$

$0.155^{*}$

0.016

$0.146^{*}$

0.024

$13.0 \quad 6.5$

$0.087^{*}$

0.036

0.220 ***

$<0.001$

12.7

8.1

$0.146^{* * *}$

$<0.001$

$0.269^{* * *}$

$<0.001$

ADAS-Cog Total Month 24

12.6

0.108

0.102

$0.195^{* * *}$

0.003

Note: $\mathrm{n}=583$ (53.0\% male); Normal $\mathrm{n}=173$ (29.7\%); MCI $\mathrm{n}=410(70.3 \%)$

GDS $=$ Geriatric Depression Scale 15-Item Version; ECog = Everyday Cognition

RAVLT = Rey Auditory Verbal Learning Test - Delayed Recall;

Trails $=$ Trail Making Test Part B;

ADAS-Cog = Alzheimer's Disease Assessment Scale-Cognitive (Inclusive Scoring)

$\mathrm{EF}=$ Executive Functioning 
Table 2

Linear Regression Model of ECog EF Predicting Logical Memory

\begin{tabular}{lcccccc}
\hline Model & $\mathbf{B}$ & S.E. & $\mathbf{t}$ & $\mathbf{S t d}-\mathbf{B}$ & $\mathbf{p}$ & $\mathbf{R}^{\mathbf{2}}$ \\
\hline Month 12 & & & & & & \\
EF Discrepancy Logical Memory & -0.042 & 0.015 & -2.768 & $\mathbf{- 0 . 0 7 7}$ & $\mathbf{0 . 0 0 6}$ & 0.570 \\
EF Subject Logical Memory & 0.015 & 0.020 & 0.750 & 0.023 & 0.453 & 0.568 \\
EF Partner Logical Memory & -0.044 & 0.018 & -2.536 & $\mathbf{- 0 . 0 7 6}$ & $\mathbf{0 . 0 1 1}$ & 0.570 \\
& & & & & & \\
Month 24 & & & & & & \\
EF Discrepancy Logical Memory & -0.036 & 0.027 & -1.324 & -0.068 & 0.187 & 0.440 \\
EF Subject Logical Memory & 0.036 & 0.034 & 1.039 & 0.055 & 0.300 & 0.438 \\
EF Partner Logical Memory & -0.018 & 0.031 & -0.569 & -0.031 & 0.570 & 0.437 \\
& & & & & & \\
\hline
\end{tabular}

Note: $\mathrm{n}=583(53.0 \%$ male $)$

Logical Memory = Logical Memory Test - Delayed Recall; ECog = Everyday Cognition All models controlled for Age, Gender, Education, Baseline GDS, and Baseline Logical Memory

$\mathrm{EF}=$ Executive Functioning 
Table 3

Linear Regression Model of ECog EF Predicting Trail Making Part B

\begin{tabular}{lcccccc}
\hline Model & & & & & & \\
\hline Month 12 & B & S.E. & $\mathbf{t}$ & Std-B & $\mathbf{p}$ & $\mathbf{R}^{\mathbf{2}}$ \\
EF Discrepancy Trails B & -0.046 & 0.162 & -0.282 & -0.007 & 0.778 & 0.624 \\
EF Subject Trails B & 0.281 & 0.216 & 1.303 & 0.037 & 0.193 & 0.625 \\
EF Partner Trails B & 0.140 & 0.181 & 0.775 & 0.021 & 0.439 & 0.624 \\
& & & & & & \\
Month 24 & & & & & & \\
EF Discrepancy Trails B & 1.022 & 0.468 & 2.185 & $\mathbf{0 . 1 2 6}$ & $\mathbf{0 . 0 3 0}$ & 0.270 \\
EF Subject Trails B & 1.091 & 0.615 & 1.774 & 0.106 & 0.077 & 0.260 \\
EF Partner Month Trails B & 1.988 & 0.500 & 3.973 & $\mathbf{0 . 2 2 8}$ & $<\mathbf{0 . 0 0 1}$ & 0.300 \\
& & & & & & \\
\hline Note: & & & & & &
\end{tabular}

Note: $\mathrm{n}=583(53.0 \%$ male $)$

Trails B $=$ Trail Making Test Part B; ECog = Everyday Cognition

All models controlled for Age, Gender, Education, Baseline GDS, and Baseline Trail Making Part B; EF = Executive Functioning 
Table 4

Linear Regression Model of ECog EF Predicting RAVLT

\begin{tabular}{lcccccc}
\hline & & & & & & \\
Model & $\mathbf{B}$ & S.E. & $\mathbf{t}$ & Std-B & $\mathbf{p}$ & $\mathbf{R}^{\mathbf{2}}$ \\
\hline Month 12 & & & & & & \\
EF Discrepancy RAVLT & -0.028 & 0.012 & -2.244 & $\mathbf{- 0 . 0 6 0}$ & $\mathbf{0 . 0 2 5}$ & 0.610 \\
EF Subject RAVLT & -0.010 & 0.016 & -0.633 & -0.018 & 0.527 & 0.605 \\
EF Partner RAVLT & -0.044 & 0.014 & -3.120 & $\mathbf{- 0 . 0 8 7}$ & $\mathbf{0 . 0 0 2}$ & 0.610 \\
& & & & & & \\
Month 24 & & & & & & \\
EF Discrepancy RAVLT & -0.032 & 0.021 & -1.521 & -0.072 & 0.130 & 0.523 \\
EF Subject RAVLT & -0.017 & 0.026 & -0.661 & -0.032 & 0.509 & 0.519 \\
EF Partner RAVLT & -0.053 & 0.023 & -2.285 & $\mathbf{- 0 . 1 0 9}$ & $\mathbf{0 . 0 2 3}$ & 0.529 \\
& & & & & & \\
\hline
\end{tabular}

Note: $\mathrm{n}=583(53.0 \%$ male);

RAVLT = Rey Auditory Verbal Learning Test - Delayed Recall;

$\mathrm{ECog}=$ Everyday Cognition;

All models controlled for Age, Gender, Education, Baseline GDS, and Baseline RAVLT $\mathrm{EF}=$ Executive Functioning 
Table 5

Linear Regression Model of ECog EF Predicting ADAS-Cog

\begin{tabular}{lcccccc}
\hline Model & B & S.E. & $\mathbf{t}$ & Std-B & $\mathbf{p}$ & $\mathbf{R}^{\mathbf{2}}$ \\
\hline Month 12 & & & & & & \\
EF Discrepancy ADAS-Cog & 0.062 & 0.020 & 3.018 & $\mathbf{0 . 0 7 6}$ & $\mathbf{0 . 0 0 3}$ & 0.650 \\
EF Subject ADAS-Cog & -0.001 & 0.027 & -0.025 & -0.001 & 0.980 & 0.645 \\
EF Partner ADAS-Cog & 0.080 & 0.023 & 3.443 & $\mathbf{0 . 0 9 1}$ & $\mathbf{0 . 0 0 1}$ & 0.650 \\
& & & & & & \\
Month 24 & & & & & & \\
EF Discrepancy ADAS-Cog & 0.055 & 0.031 & 1.806 & 0.074 & 0.072 & 0.640 \\
EF Subject ADAS-Cog & 0.001 & 0.037 & 0.002 & 0.039 & 0.969 & 0.630 \\
EF Partner ADAS-Cog & 0.069 & 0.034 & 2.046 & $\mathbf{0 . 0 8 7}$ & $\mathbf{0 . 0 4 2}$ & 0.650 \\
& & & & & & \\
\hline Note:n & & & & & &
\end{tabular}

Note: $\mathrm{n}=583(53.0 \%$ male $)$

ADAS-Cog = Alzheimer's Disease Assessment Scale-Cognitive (Inclusive Scoring); ECog = Everyday Cognition; EF = Executive Functioning

All models controlled for Age, Gender, Education, Baseline GDS, and Baseline ADAS-Cog; 
Table 6

Linear Regression Model of ECog EF Predicting Clock Drawing

\begin{tabular}{lcccccc}
\hline Model & B & S.E. & $\mathbf{t}$ & Std-B & $\mathbf{p}$ & $\mathbf{R}^{\mathbf{2}}$ \\
\hline Month 12 & & & & & & \\
EF Discrepancy Clock Drawing & -0.003 & 0.003 & -0.922 & -0.034 & 0.357 & 0.224 \\
EF Subject Clock Drawing & 0.001 & 0.004 & 0.190 & 0.008 & 0.849 & 0.231 \\
EF Partner Clock Drawing & -0.003 & 0.003 & -0.872 & -0.034 & 0.383 & 0.232 \\
& & & & & & \\
Month 24 & & & & & & \\
EF Discrepancy Clock Drawing & $<0.001$ & 0.004 & -0.110 & 0.007 & 0.912 & 0.144 \\
EF Subject Clock Drawing & $<0.001$ & 0.006 & 0.019 & 0.001 & 0.985 & 0.144 \\
EF Partner Clock Drawing & -0.001 & 0.005 & -0.106 & -0.007 & 0.916 & 0.144 \\
& & & & & & \\
\hline Note: n- & & & & & &
\end{tabular}

Note: $\mathrm{n}=583(53.0 \%$ male $)$

All models controlled for Age, Gender, Education, Baseline GDS, and Baseline Clock Drawing; ECog = Everyday Cognition; EF = Executive Functioning 
Table 7

Logistic Regression Models of ECog EF Predicting Diagnostic Conversion

\begin{tabular}{|c|c|c|c|c|c|c|c|}
\hline Model & B & S.E. & Wald & df & $\operatorname{Exp}(B)$ & $\mathbf{p}$ & $\mathbf{R}^{2}$ \\
\hline \multicolumn{8}{|l|}{ Month 12} \\
\hline EF Discrepancy Diagnostic Conversion & 0.044 & 0.015 & 8.929 & 1 & 1.045 & 0.003 & 0.020 \\
\hline EF Subject Diagnostic Conversion & 0.038 & 0.018 & 4.738 & 1 & 1.039 & $\mathbf{0 . 0 3 0}$ & 0.020 \\
\hline EF Partner Diagnostic Conversion & 0.067 & 0.014 & 23.974 & 1 & 1.069 & $<0.001$ & 0.044 \\
\hline \multicolumn{8}{|l|}{ Month 24} \\
\hline EF Discrepancy Diagnostic Conversion & 0.025 & 0.019 & 1.807 & 1 & 1.025 & 0.179 & 0.031 \\
\hline EF Subject Diagnostic Conversion & 0.015 & 0.025 & 0.372 & 1 & 1.015 & 0.542 & 0.020 \\
\hline EF Partner Diagnostic Conversion & 0.035 & 0.018 & 3.686 & 1 & 1.036 & 0.055 & 0.038 \\
\hline
\end{tabular}

Note: $\mathrm{n}=583$ (53.0\% male); Normal $\mathrm{n}=173$ (29.7\%); MCI $\mathrm{n}=410(70.3 \%)$

All models controlled for Age, Gender, Education and Baseline GDS.

ECog $=$ Everyday Cognition $; \mathrm{EF}=$ Executive Functioning 
Table 8

Study Sample Characteristics and Correlations with ECog Scales - All Subjects

\begin{tabular}{|c|c|c|c|c|c|c|}
\hline Variable & Mean & $\mathrm{SD}$ & $\begin{array}{l}\text { EF Disc } \\
\text { Pearson }\end{array}$ & $\begin{array}{l}\text { EF Disc } \\
\text { p-value }\end{array}$ & $\begin{array}{l}\text { Memory } \\
\text { Disc } \\
\text { Pearson }\end{array}$ & $\begin{array}{c}\text { Memory } \\
\text { Disc } \\
\text { p-value }\end{array}$ \\
\hline Age at Baseline & 72.5 & 7.3 & $0.080 *$ & 0.039 & $0.145 * *$ & $<0.001$ \\
\hline Education (years) & 16.3 & 2.6 & $-0.099 *$ & 0.011 & -0.073 & 0.060 \\
\hline GDS-Baseline & 1.5 & 1.5 & -0.071 & 0.070 & -0.045 & 0.244 \\
\hline ECog EF Subject & 23.0 & 8.5 & $-0.430 * *$ & $<0.001$ & $-0.244 * *$ & $<0.001$ \\
\hline ECog Memory Subject & 16.9 & 5.9 & $-0.179 * *$ & $<0.001$ & $-0.384 * *$ & $<0.001$ \\
\hline ECog EF Study Partner & 24.0 & 11.5 & $0.745 * *$ & $<0.001$ & $0.586 * *$ & $<0.001$ \\
\hline ECog Memory Study Partner & 16.3 & 7.4 & $0.569 * *$ & $<0.001$ & $0.682 * *$ & $<0.001$ \\
\hline ECog EF Discrepancy & 1.0 & 12.2 & . & . & $0.721 * *$ & $<0.001$ \\
\hline ECog Memory Discrepancy & -0.5 & 7.3 & $0.721 * *$ & $<0.001$ & . & . \\
\hline Logical Memory Baseline & 8.2 & 4.7 & $-0.335 * *$ & $<0.001$ & $-0.345 * *$ & $<0.001$ \\
\hline Logical Memory Month 12 & 8.9 & 5.8 & $-0.350 * *$ & $<0.001$ & $-0.366^{* * *}$ & $<0.001$ \\
\hline Logical Memory Month 24 & 9.7 & 5.6 & $-0.249 * *$ & $<0.001$ & $-0.281 * *$ & $<0.001$ \\
\hline RAVLT Delayed Baseline & 5.1 & 4.3 & $-0.293 * *$ & $<0.001$ & $-0.338 * *$ & $<0.001$ \\
\hline RAVLT Delayed Month 12 & 5.0 & 4.7 & $-0.281 * *$ & $<0.001$ & $-0.325 * *$ & $<0.001$ \\
\hline RAVLT Delayed Month 24 & 4.8 & 4.5 & $-0.243 * *$ & $<0.001$ & $-0.236 * *$ & $<0.001$ \\
\hline Clock Drawing Baseline & 4.4 & 0.9 & $-0.250 * *$ & $<0.001$ & $-0.264 * *$ & $<0.001$ \\
\hline Clock Drawing Month 12 & 4.4 & 1.0 & $-0.225 * *$ & $<0.001$ & $-0.239 * *$ & $<0.001$ \\
\hline Clock Drawing Month 24 & 4.5 & 0.9 & $-0.167 * *$ & 0.008 & $-0.161 *$ & 0.010 \\
\hline Trails Baseline & 107.4 & 65.0 & $0.271 * *$ & $<0.001$ & $0.307 * *$ & $<0.001$ \\
\hline Trails Month 12 & 111.8 & 72.6 & $0.255 * *$ & $<0.001$ & $0.305 * *$ & $<0.001$ \\
\hline Trails Month 24 & 103.9 & 82.6 & $0.174 * *$ & 0.006 & $0.187 * *$ & 0.003 \\
\hline ADAS-Cog Total Baseline & 15.0 & 8.7 & $0.377 * *$ & $<0.001$ & $0.407 * *$ & $<0.001$ \\
\hline ADAS-Cog Total Month 12 & 15.2 & 10.9 & $0.410 * *$ & $<0.001$ & $0.426 * *$ & $<0.001$ \\
\hline ADAS-Cog Total Month 24 & 14.3 & 10.2 & $0.306 * *$ & $<0.001$ & $0.325 * *$ & $<0.001$ \\
\hline
\end{tabular}

Note: $\mathrm{n}=661$ (53.7\% male); Normal $\mathrm{n}=173(26.2 \%)$; MCI $\mathrm{n}=410(62.0 \%) ;$ AD $\mathrm{n}=78(11.8 \%)$

GDS $=$ Geriatric Depression Scale 15-Item Version; ECog = Everyday Cognition

RAVLT $=$ Rey Auditory Verbal Learning Test - Delayed Recall; Trails = Trail Making Test Part B;

ADAS-Cog = Alzheimer's Disease Assessment Scale-Cognitive (Inclusive Scoring)

Logical Memory = Logical Memory Test - Delayed Recall 
Table 9

Path Model Comparisons of ECog EF Prediction

\begin{tabular}{lcc} 
Model & $\boldsymbol{\chi 2} / \mathbf{d f}$ & $\mathbf{p}$ \\
\hline Month 12 & & 0.475 \\
Trail Making Test Part B & 0.510 & 0.853 \\
Alzheimer's Disease Assessment Scale-Cognitive & 0.034 & 0.097 \\
Rey Auditory Verbal Learning Test - Delayed Recall & 2.762 & \\
Month 24 & & 0.200 \\
Trail Making Test Part B & 1.645 & 0.553 \\
Alzheimer's Disease Assessment Scale-Cognitive & 0.352 & 0.369 \\
Rey Auditory Verbal Learning Test - Delayed Recall & 0.809 & \\
\hline
\end{tabular}

Note: $\mathrm{n}=661(53.7 \%$ male $)$

Month 12 Normal Memory $n=444$, Impaired Memory $n=217$

Month 24 Normal Memory $n=202$, Impaired Memory $n=45$

$\mathrm{ECog}=$ Everyday Cognition; $\mathrm{EF}=$ Executive Functioning 
Table 10

Linear Regression Model of ECog Memory Predicting Logical Memory

\begin{tabular}{lcccccc}
\hline & & & & & & \\
Model & $\mathbf{B}$ & S.E. & $\mathbf{t}$ & Std-B & $\mathbf{p}$ & $\mathbf{R}^{\mathbf{2}}$ \\
\hline Month 12 & & & & & & \\
Memory Discrepancy Logical Memory & -0.074 & 0.023 & -3.177 & $\mathbf{- 0 . 0 8 9}$ & $\mathbf{0 . 0 0 2}$ & 0.580 \\
Memory Subject Logical Memory & -0.044 & 0.030 & -1.475 & -0.046 & 0.141 & 0.569 \\
Memory Partner Logical Memory & -0.142 & 0.027 & -5.215 & $\mathbf{- 0 . 1 6 9}$ & $<\mathbf{0 . 0 0 1}$ & 0.590 \\
& & & & & & \\
Month 24 & & & & & & \\
Memory Discrepancy Logical Memory & -0.082 & 0.041 & -2.012 & $\mathbf{- 0 . 1 0 3}$ & $\mathbf{0 . 0 4 5}$ & 0.446 \\
Memory Subject Logical Memory & -0.022 & 0.050 & -0.436 & -0.024 & 0.663 & 0.436 \\
Memory Partner Logical Memory & -0.136 & 0.048 & -2.845 & $\mathbf{- 0 . 1 6 0}$ & $\mathbf{0 . 0 0 5}$ & 0.455 \\
& & & & & & \\
\hline
\end{tabular}

Note: $\mathrm{n}=583(53.0 \%$ male $)$

Logical Memory $=$ Logical Memory Test - Delayed Recall; ECog = Everyday Cognition All models controlled for Age, Gender, Education, Baseline GDS, and Baseline Logical Memory 
Table 11

Linear Regression Model of ECog Memory Predicting Trail Making Part B

\begin{tabular}{lcccccc}
\hline & & & & & & \\
Model & B & S.E. & $\mathbf{t}$ & Std-B & $\mathbf{p}$ & $\mathbf{R}^{\mathbf{2}}$ \\
\hline Month 12 & & & & & & \\
Memory Discrepancy Trails B & 0.207 & 0.249 & 0.831 & 0.022 & 0.406 & 0.624 \\
Memory Subject Trails B & 0.385 & 0.380 & 1.014 & 0.036 & 0.311 & 0.625 \\
Memory Partner Trails B & 0.615 & 0.265 & 2.319 & $\mathbf{0 . 0 6 5}$ & $\mathbf{0 . 0 2 1}$ & 0.627 \\
& & & & & & \\
Month 24 & & & & & & \\
Memory Discrepancy Trails B & 1.059 & 0.702 & 1.509 & 0.088 & 0.133 & 0.259 \\
Memory Subject Trails B & 1.520 & 0.840 & 1.810 & 0.108 & 0.072 & 0.300 \\
Memory Partner Trails B & 2.483 & 0.746 & 3.328 & $\mathbf{0 . 1 9 4}$ & $\mathbf{0 . 0 0 1}$ & 0.290 \\
& & & & & & \\
\hline
\end{tabular}

Note: $\mathrm{n}=583(53.0 \%$ male $)$

Trails B = Trail Making Test Part B; ECog = Everyday Cognition

All models controlled for Age, Gender, Education, Baseline GDS, and Baseline Trail Making Part B; 
Table 12

Linear Regression Model of ECog Memory Predicting RAVLT

\begin{tabular}{lcccccc}
\hline Model & $\mathbf{B}$ & S.E. & $\mathbf{t}$ & $\mathbf{S t d - B}$ & $\mathbf{p}$ & $\mathbf{R}^{\mathbf{2}}$ \\
\hline Month 12 & & & & & & \\
Memory Discrepancy RAVLT & -0.048 & 0.019 & -2.495 & $\mathbf{- 0 . 0 6 7}$ & $\mathbf{0 . 0 1 3}$ & 0.609 \\
Memory Subject RAVLT & -0.038 & 0.023 & -1.624 & -0.047 & 0.105 & 0.607 \\
Memory Partner RAVLT & -0.091 & 0.021 & -4.301 & $\mathbf{- 0 . 1 2 6}$ & $<\mathbf{0 . 0 0 1}$ & 0.620 \\
& & & & & & \\
Month 24 & & & & & & \\
Memory Discrepancy RAVLT & -0.041 & 0.031 & -1.318 & -0.063 & 0.189 & 0.522 \\
Memory Subject RAVLT & -0.065 & 0.037 & -1.751 & -0.085 & 0.081 & 0.525 \\
Memory Partner RAVLT & -0.110 & 0.035 & -3.191 & $\mathbf{- 0 . 1 5 6}$ & $\mathbf{0 . 0 0 2}$ & 0.539 \\
& & & & & & \\
\hline
\end{tabular}

Note: $\mathrm{n}=583(53.0 \%$ male $)$

RAVLT = Rey Auditory Verbal Learning Test - Delayed Recall;

ECog = Everyday Cognition

All models controlled for Age, Gender, Education, Baseline GDS, and Baseline RAVLT 
Table 13

Linear Regression Model of ECog Memory Predicting ADAS-Cog

\begin{tabular}{lcccccc}
\hline Model & & & & & & \\
\hline Month 12 & B & S.E. & $\mathbf{t}$ & Std-B & $\mathbf{p}$ & $\mathbf{R}^{\mathbf{2}}$ \\
Memory Discrepancy ADASCog & 0.120 & 0.032 & 3.770 & $\mathbf{0 . 0 9 6}$ & $<\mathbf{0 . 0 0 1}$ & 0.650 \\
Memory Subject ADASCog & $<0.001$ & 0.040 & -0.012 & $<0.001$ & 0.990 & 0.645 \\
Memory Partner ADASCog & 0.154 & 0.036 & 4.280 & $\mathbf{0 . 1 2 3}$ & $<\mathbf{0 . 0 0 1}$ & 0.660 \\
& & & & & & \\
Month 24 & & & & & & \\
Memory Discrepancy ADAS-Cog & 0.120 & 0.045 & 2.678 & $\mathbf{0 . 1 1 0}$ & $\mathbf{0 . 0 0 8}$ & 0.640 \\
Memory Subject ADAS-Cog & 0.088 & 0.055 & 1.614 & 0.071 & 0.108 & 0.634 \\
Memory Partner ADAS-Cog & 0.232 & 0.049 & 4.710 & $\mathbf{0 . 1 9 9}$ & $<\mathbf{0 . 0 0 1}$ & 0.660 \\
& & & & & & \\
\hline
\end{tabular}

Note: $\mathrm{n}=583(53.0 \%$ male $)$

ADAS-Cog = Alzheimer's Disease Assessment Scale-Cognitive (Inclusive Scoring);

ECog $=$ Everyday Cognition

All models controlled for Age, Gender, Education, Baseline GDS, and Baseline ADAS-Cog 
Table 14

Linear Regression Model of ECog Memory Predicting Clock Drawing

\begin{tabular}{lcccccc}
\hline Model & & & & & & \\
\hline Month 12 & B & S.E. & $\mathbf{t}$ & Std-B & $\mathbf{p}$ & $\mathbf{R}^{\mathbf{2}}$ \\
Memory Discrepancy Clock Drawing & -0.010 & 0.004 & -2.178 & $-\mathbf{0 . 0 8 1}$ & $\mathbf{0 . 0 3 0}$ & 0.237 \\
Memory Subject Clock Drawing & -0.003 & 0.005 & -0.507 & -0.020 & 0.612 & 0.231 \\
Memory Partner Clock Drawing & -0.013 & 0.005 & -2.739 & $\mathbf{- 0 . 1 0 6}$ & $\mathbf{0 . 0 0 6}$ & 0.241 \\
& & & & & & \\
Month 24 & & & & & & \\
Memory Discrepancy Clock Drawing & -0.001 & 0.007 & -0.144 & -0.009 & 0.885 & 0.144 \\
Memory Subject Clock Drawing & -0.010 & 0.008 & -1.311 & -0.084 & 0.191 & 0.151 \\
Memory Partner Clock Drawing & -0.010 & 0.007 & -1.341 & -0.084 & 0.181 & 0.151 \\
& & & & & & \\
\hline
\end{tabular}

Note: $\mathrm{n}=583(53.0 \%$ male $)$

All models controlled for Age, Gender, Education, Baseline GDS, and Baseline Clock Drawing; ECog $=$ Everyday Cognition 
Table 15

Logistic Regression Models of ECog Memory Predicting Diagnostic Conversion

\begin{tabular}{|c|c|c|c|c|c|c|c|}
\hline Model & B & S.E. & Wald & df & $\operatorname{Exp}(B)$ & $\mathbf{p}$ & $\mathbf{R}^{2}$ \\
\hline \multicolumn{8}{|l|}{ Month 12} \\
\hline Memory Discrepancy Diagnostic Conversion & 0.100 & 0.024 & 16.854 & 1 & 1.105 & $<0.001$ & 0.040 \\
\hline Memory Subject Diagnostic Conversion & 0.061 & 0.028 & 4.720 & 1 & 1.063 & $\mathbf{0 . 0 3 0}$ & 0.020 \\
\hline Memory Partner Diagnostic Conversion & 0.140 & 0.024 & 33.501 & 1 & 1.150 & $<0.001$ & 0.070 \\
\hline \multicolumn{8}{|l|}{ Month 24} \\
\hline Memory Discrepancy Diagnostic Conversion & 0.068 & 0.031 & 4.863 & 1 & 1.070 & 0.027 & 0.050 \\
\hline Memory Subject Diagnostic Conversion & 0.033 & 0.034 & 0.912 & 1 & 1.033 & 0.339 & 0.030 \\
\hline Memory Partner Diagnostic Conversion & 0.097 & 0.031 & 10.064 & 1 & 1.102 & 0.002 & 0.070 \\
\hline
\end{tabular}

Note: $\mathrm{n}=583(53.0 \%$ male $)$

All models controlled for Age, Gender, Education and Baseline GDS.

$\mathrm{ECog}=$ Everyday Cognition 
Table 16

Linear and Logistic Regression Models of Simultaneous ECog EF and Memory Prediction

\begin{tabular}{|c|c|c|c|c|c|}
\hline \multirow[b]{2}{*}{ Model } & \multicolumn{2}{|c|}{ Executive Functioning } & \multicolumn{2}{|c|}{ Memory } & \multirow[b]{2}{*}{$\mathbf{R}^{2}$} \\
\hline & Std-B & $\mathbf{p}$ & Std-B & $\mathbf{p}$ & \\
\hline Month 12 Logical Memory & -0.035 & 0.336 & -0.067 & 0.069 & 0.580 \\
\hline Month 24 Logical Memory & -0.002 & 0.975 & -0.102 & 0.133 & 0.446 \\
\hline Month 12 Trails B & -0.037 & 0.281 & 0.046 & 0.183 & 0.625 \\
\hline Month 24 Trails B & 0.121 & 0.117 & 0.009 & 0.911 & 0.270 \\
\hline Month 12 RAVLT & -0.029 & 0.399 & -0.048 & 0.170 & 0.610 \\
\hline Month 24 RAVLT & -0.054 & 0.385 & -0.027 & 0.664 & 0.524 \\
\hline Month 12 ADAS-Cog & 0.025 & 0.438 & 0.079 & 0.018 & 0.650 \\
\hline Month 24 ADAS-Cog & 0.007 & 0.889 & 0.105 & 0.051 & 0.640 \\
\hline Month 12 Clock & 0.031 & 0.521 & -0.101 & $\mathbf{0 . 0 3 9}$ & 0.238 \\
\hline Month 24 Clock & -0.002 & 0.985 & -0.008 & 0.924 & 0.144 \\
\hline Month 12 Diagnostic Conversion (Logistic) & 1.003 & 0.889 & 1.101 & 0.004 & 0.040 \\
\hline Month 24 Diagnostic Conversion (Logistic) & 0.992 & 0.769 & 1.080 & 0.075 & 0.050 \\
\hline
\end{tabular}

Note: $n=583$ (53.0\% male); Normal $\mathrm{n}=173$ (29.7\%); MCI $\mathrm{n}=410(70.3 \%)$

All models including both baseline executive functioning and memory discrepancy scores as covariates.

GDS $=$ Geriatric Depression Scale 15-Item Version; ECog = Everyday Cognition Scale

RAVLT = Rey Auditory Verbal Learning Test - Delayed Recall; Trails = Trail Making Test Part B;

ADAS-Cog = Alzheimer's Disease Assessment Scale-Cognitive (Inclusive Scoring) 


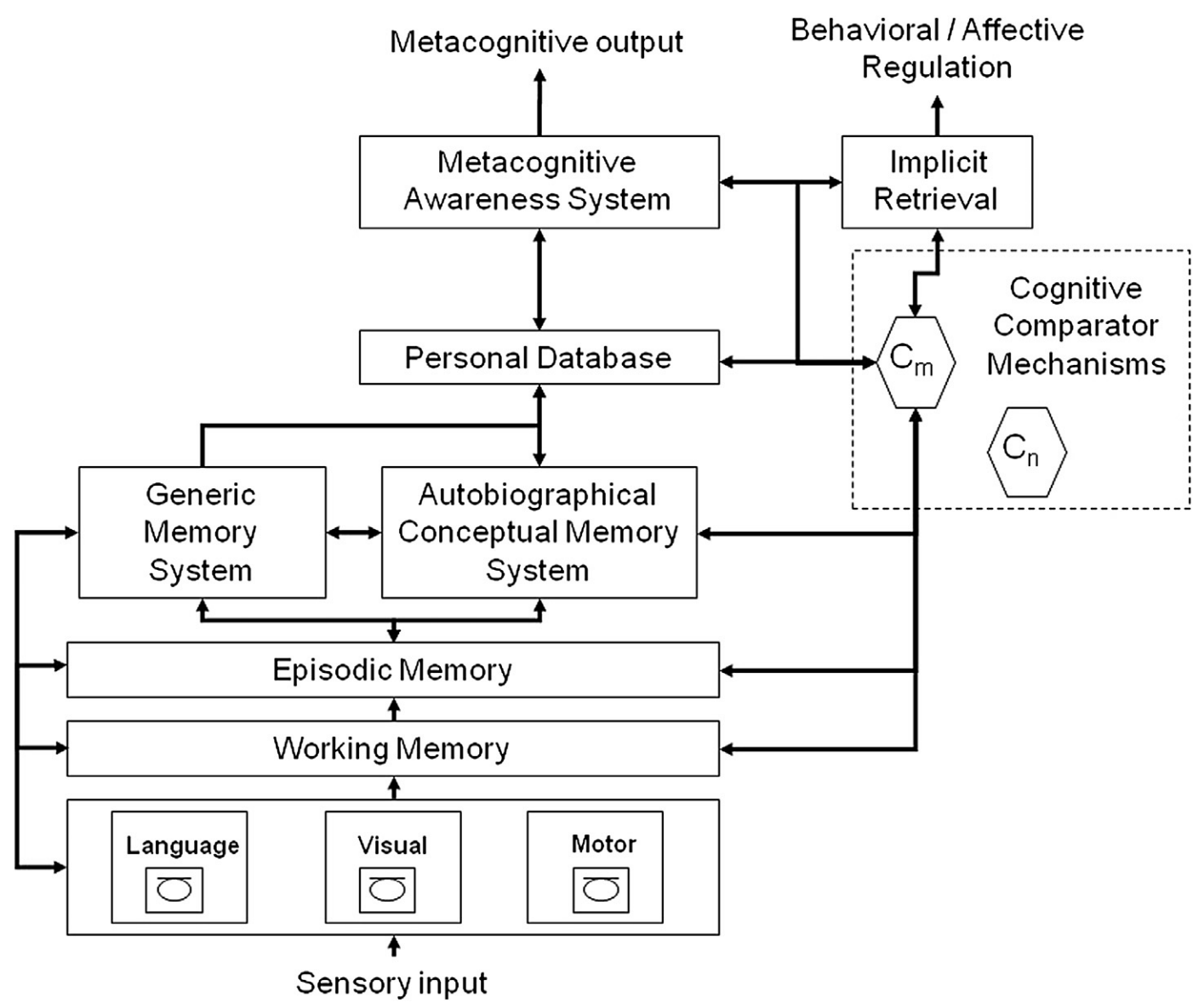

Figure 1. Cognitive Awareness Model (CAM; Morris \& Mograbi, 2013) 


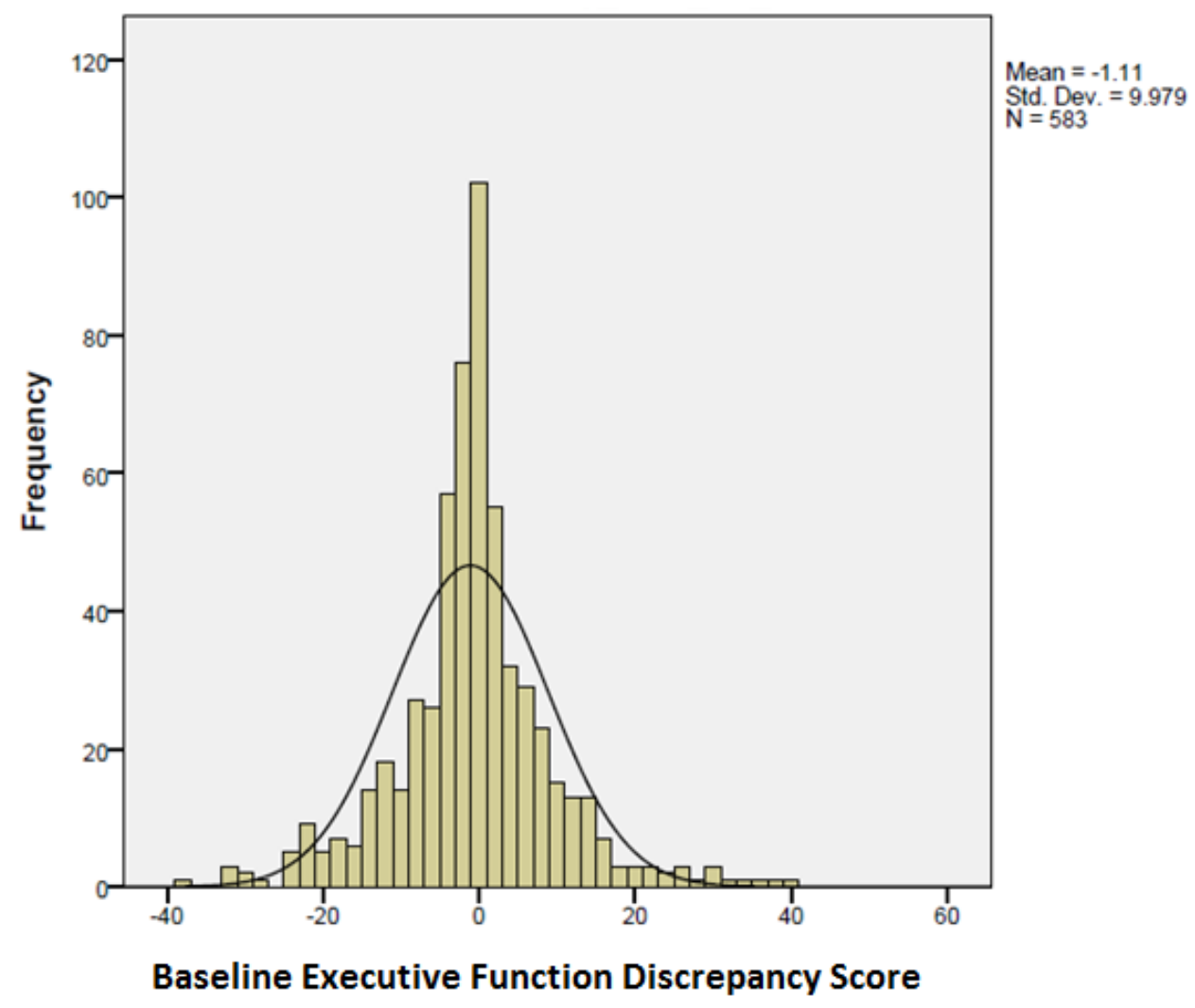

Figure 2. Histogram of normal and MCI executive functioning discrepancy scores. 


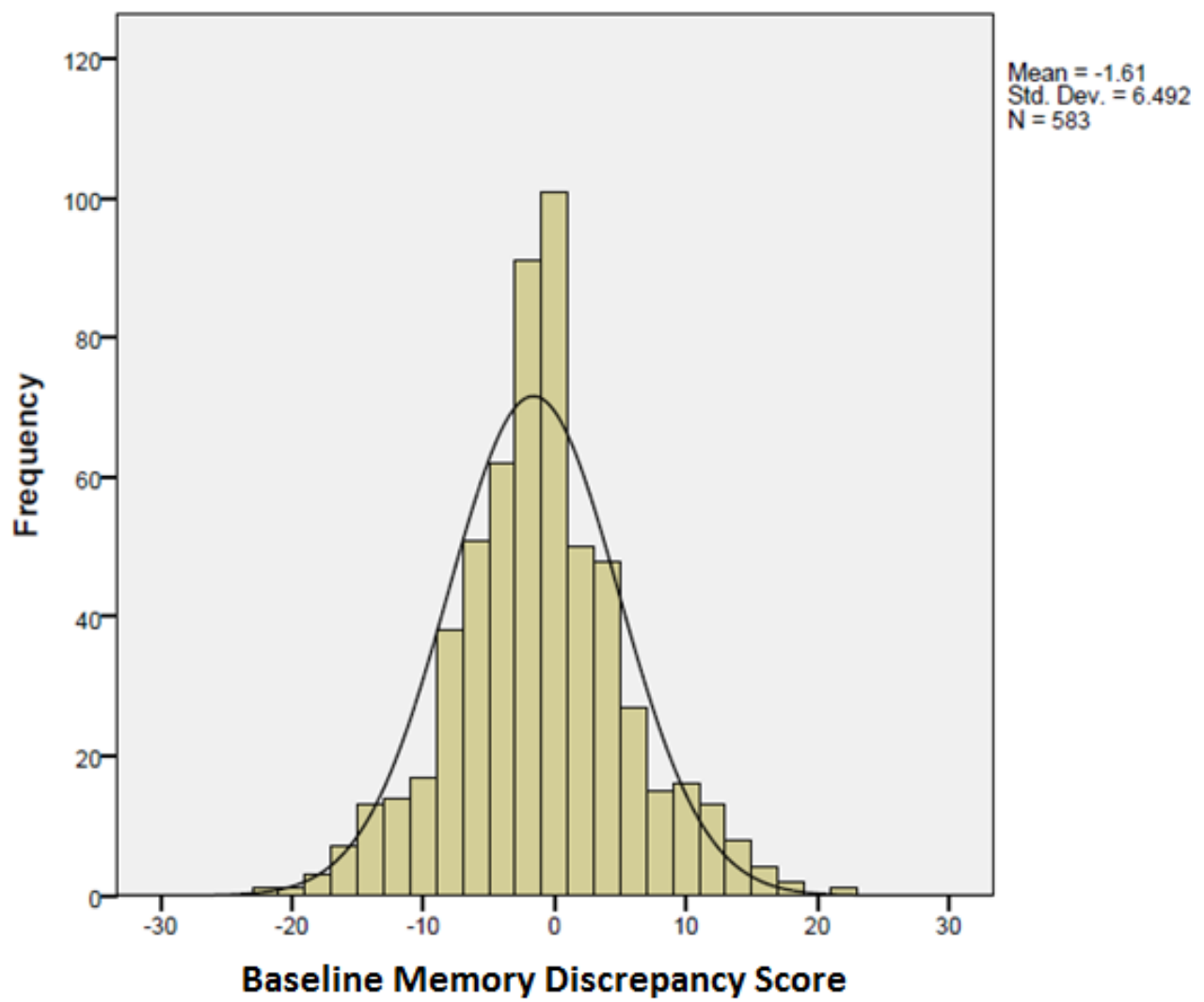

Figure 3. Histogram of normal and MCI memory discrepancy scores. 


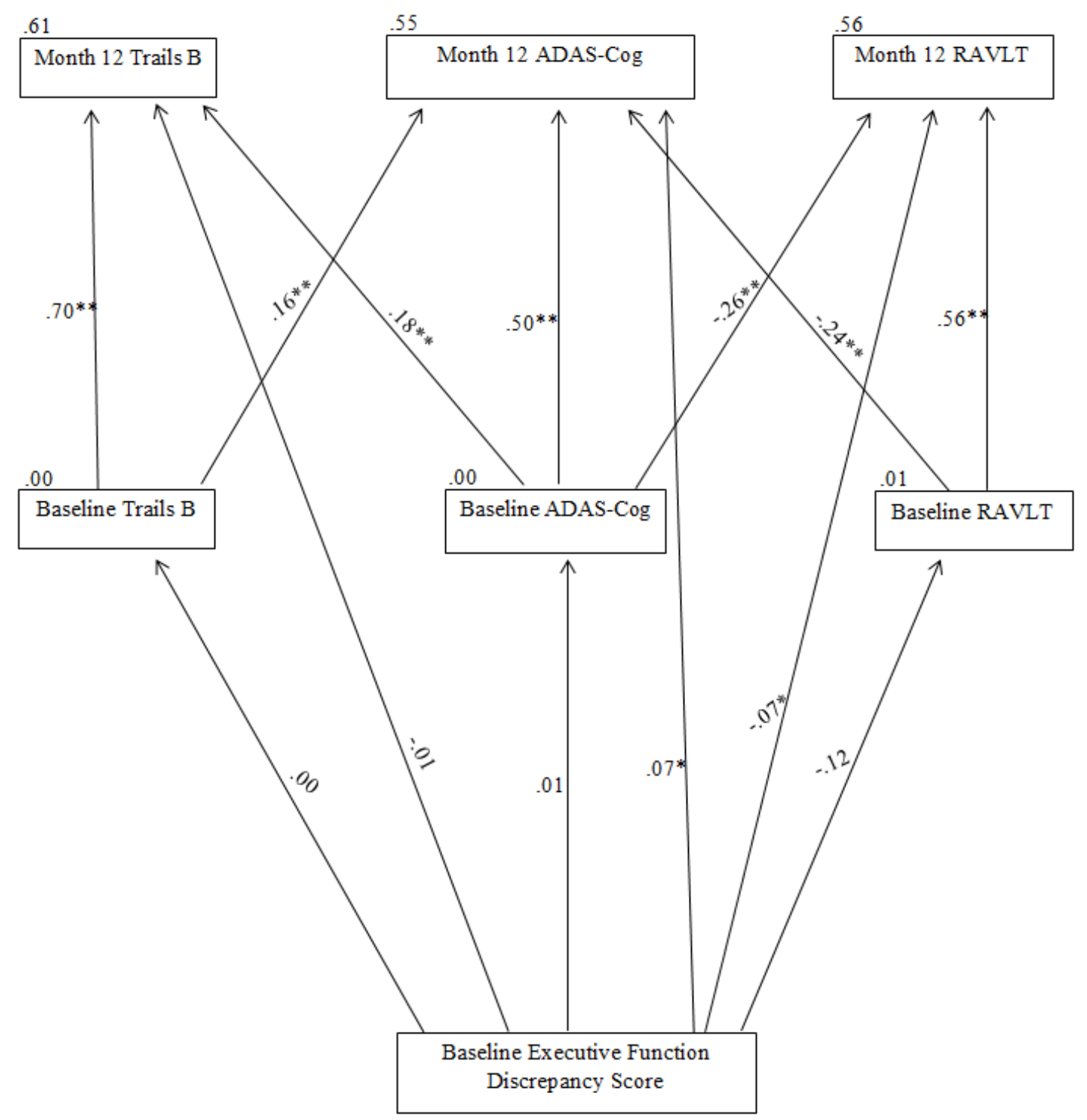

Figure 4. Month12 normal memory model of ECog executive functioning discrepancy prediction. Values are standardized. $\mathrm{n}=444$. $^{*}=\mathrm{p}<0.05, * *=\mathrm{p}<0.01$.

Not shown: all errors terms at baseline were correlated and all error terms at follow-up were correlated. 


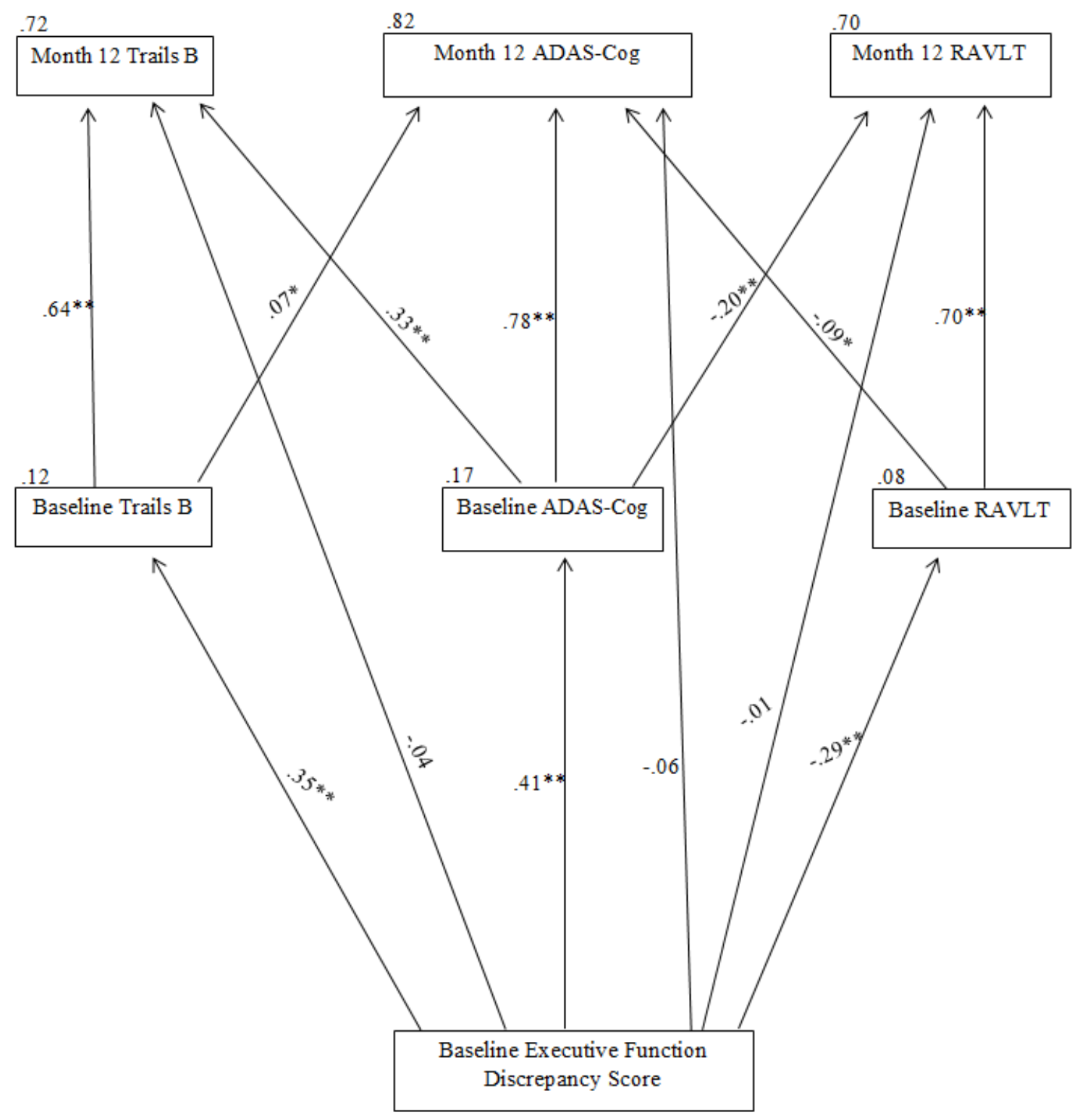

Figure 5. Month 12 impaired memory model of ECog executive functioning discrepancy prediction. Values are standardized. $\mathrm{n}=217 . * \mathrm{p}<0.05, * *=\mathrm{p}<0.01$.

Not shown: all errors terms at baseline were correlated and all error terms at follow-up were correlated. 


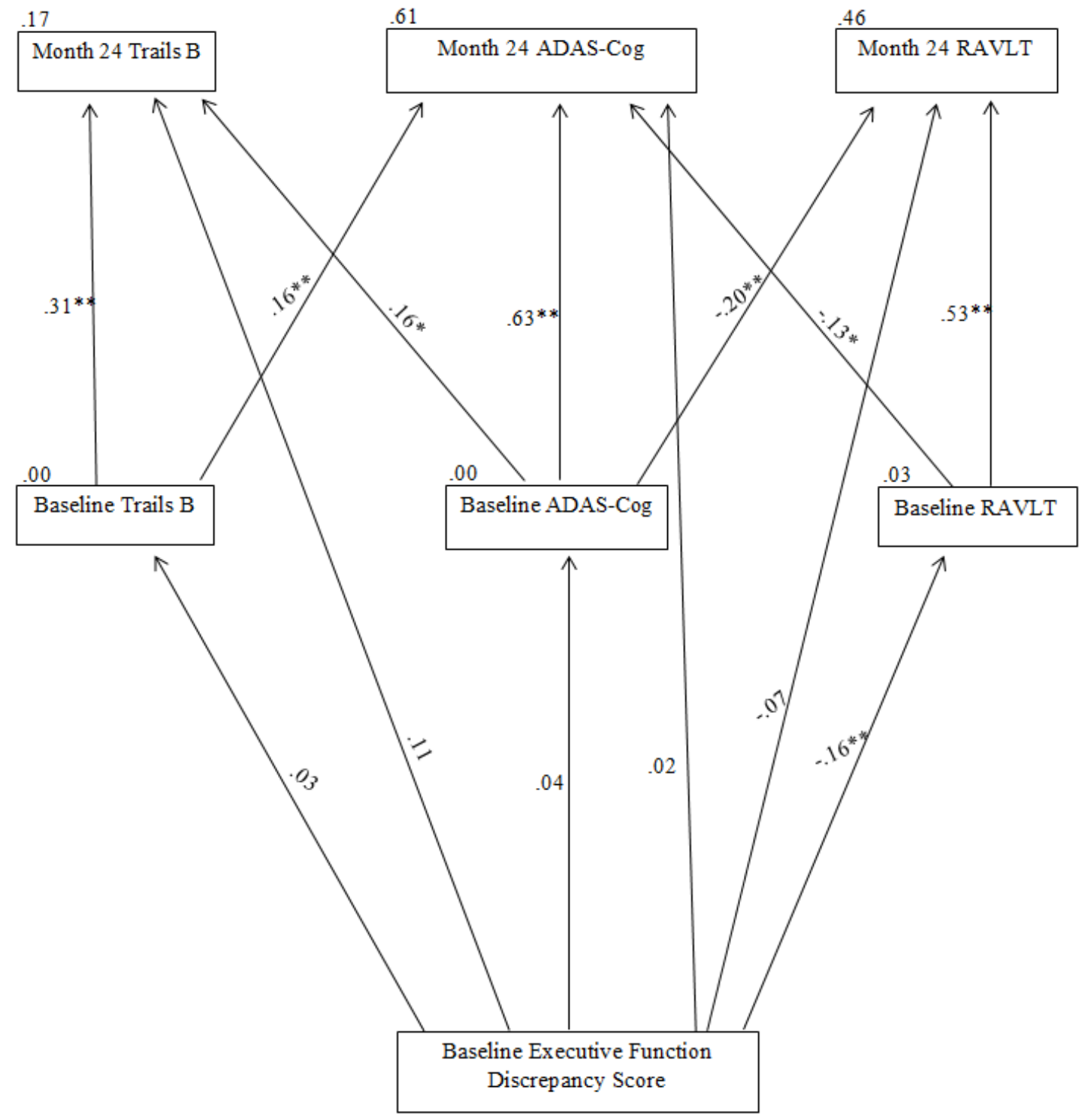

Figure 6. Month 24 normal memory model of ECog executive functioning discrepancy prediction. Values are standardized. $\mathrm{n}=202 . *=\mathrm{p}<0.05, * *=\mathrm{p}<0.01$.

Not shown: all errors terms at baseline were correlated and all error terms at follow-up were correlated. 


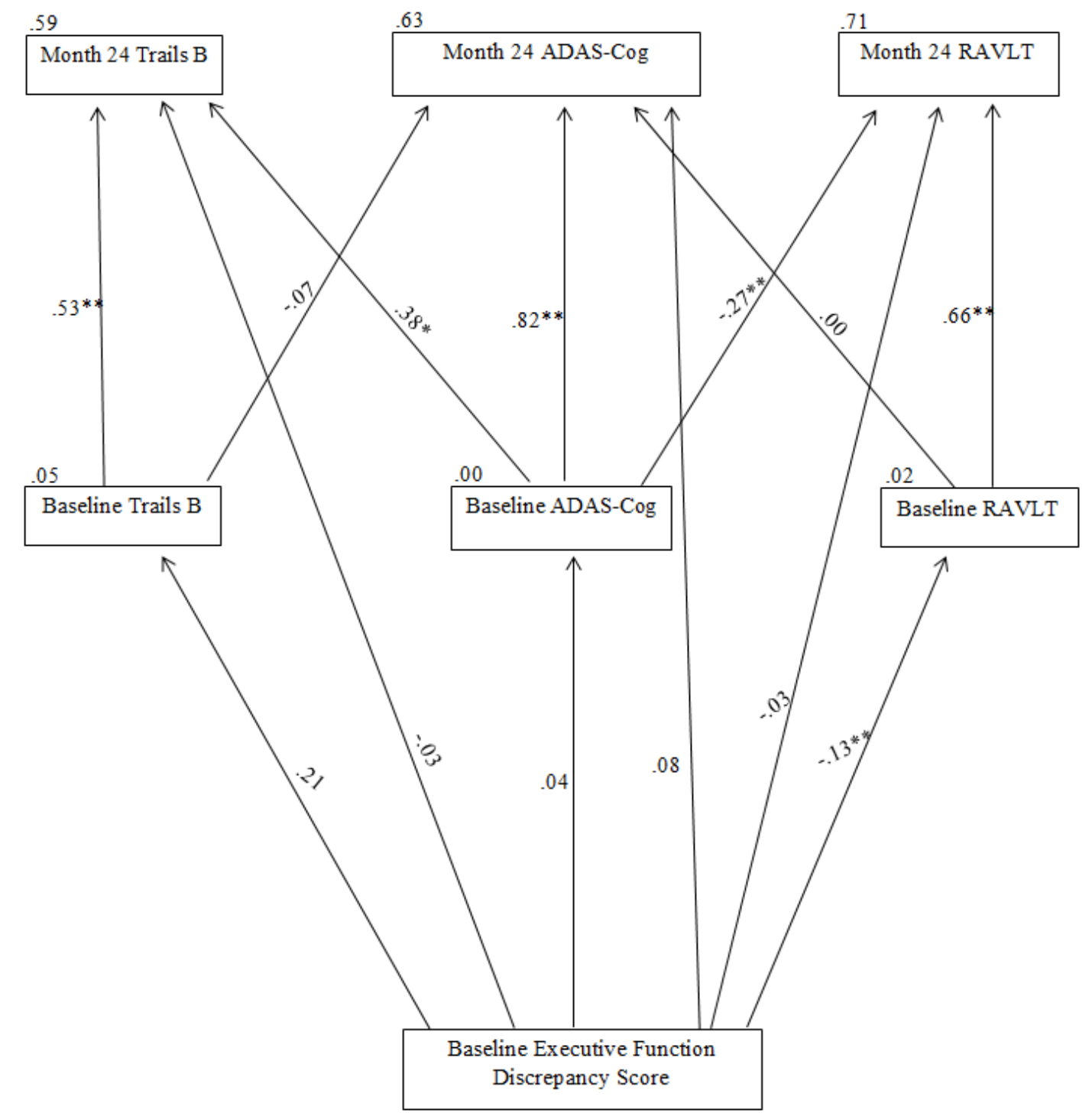

Figure 7. Month 24 impaired memory model of ECog executive functioning discrepancy prediction. Values are standardized. $\mathrm{n}=45$. $^{*}=\mathrm{p}<0.05$, $* *=\mathrm{p}<0.01$.

Not shown: all errors terms at baseline were correlated and all error terms at follow-up were correlated. 


\title{
CURRICULUM VITA
}

\author{
Jeremy Stark Carmasin \\ University of Louisville - Department of Psychological and Brain Sciences \\ Life Sciences 123 \\ Louisville, KY, 40292
}

\section{EDUCATION}

\section{University of Louisville, Louisville, KY}

Mentor: Benjamin Mast, Ph.D.

Ph.D. Candidate in Clinical Psychology (degree expected August 2015)

Dissertation: Awareness of Executive Functioning as a Diagnostic Tool for Mild Cognitive Impairment and Alzheimer's Disease

M.A. in Clinical Psychology, May 2013

\section{Brandeis University, Waltham, MA}

B.A. in Psychology cum laude, May 2007

Minors: Computer Science, Internet Studies, Theater Arts

Academic Honors: Dean's List, Psi Chi National Honor Society

\author{
AFFILIATION \\ American Psychological Association (APA) \\ September 2011 - Present \\ Gerontological Society of America (GSA) \\ September 2010 - Present
}

\section{CLINICAL EXPERIENCE}

\section{Robley Rex VA Medical Center, Louisville, KY}

August 2013 - June 2014

Neuropsychological Assessment. Supervisor: Marilyn Wagner, Ph.D.

-Administered clinical interviews and neuropsychological testing to veterans.

Wrote integrated reports.

-Reviewed medical chart data such as combat history, medication use, and previous psychological testing. 


\section{University of Louisville Psychological Services Center, Louisville, KY}

August 2012 - June 2014

Mindfulness Practice. Supervisor: Paul Salmon, Ph.D.

-Administered mindfulness-focused therapy to clients.

-Supervised fellow student therapists at weekly meetings.

\section{University of Louisville Psychological Services Center, Louisville, KY}

May 2012 - June 2014

Clinical Assistant. Supervisor: Bernadette Walter, Ph.D.

-Brought new clients into treatment via brief phone intakes and detailed in-person clinical interviews.

-Assisted in determining clinical team placement.

-Maintained client records, budgets, and clinical reports.

\section{Private Practice of Steven J. Simon, Louisville, KY}

May 2012 - August 2013

Disability Assessment. Supervisor: Steven J. Simon, Ph.D.

-Administered clinical interviews, psychodiagnostic measures, and neuropsychological testing measures.

-Wrote integrated reports for adults with a variety of impairments.

\section{University of Louisville Psychological Services Center, Louisville, KY}

May 2011 - June 2014

Intellectual Ability and Disability Assessment. Supervisor: Bernadette Walter, Ph.D.

-Administered clinical interviews, psychodiagnostic measures, and neuropsychological testing measures.

-Wrote integrated reports for children and adults.

\section{University of Louisville Geriatric Clinic, Louisville, KY}

August 2010 - August 2012

Geriatric Assessment. Supervisor: Benjamin Mast, Ph.D.

-Administered clinical interviews and neuropsychological testing to older adults. Wrote integrated reports.

-Collaborated with interdisciplinary team in diagnosis and treatment.

-Reviewed medical chart data such as blood labs and MRI.

-Administered free memory screenings to community-dwelling older adults.

-Supervised fellow student therapists at weekly meetings. 


\section{TEACHING EXPERIENCE}

\section{University of Louisville Psychological Services Center, Louisville, KY}

May 2012 - June 2014

Clinical Assistant

-Instructed and supervised other graduate students in clinical interviewing and assessment coursework.

\section{University of Louisville, Louisville, KY}

August 2010 - May 2012

Graduate Teaching Assistant

-Assisted faculty in coursework for Introductory, Experimental, and Forensic

Psychology.

-Responsibilities included teaching class lectures and lab sections, test preparation, and grading.

\section{RESEARCH EXPERIENCE}

\section{University of Louisville, Louisville, KY}

July 2010 - Present

Graduate Research Assistant/Coordinator. Supervisor: Benjamin Mast, Ph.D.

Baltimore Study of Black Aging (BSBA):

-Analyzed longitudinal data, including neuropsychological, demographic, and self-report measures.

Study of Thinking and Relationships (STAR):

-Gathered and analyzed neuropsychological and self-report data for community-dwelling older adults.

-Oversaw recruitment and data collection by undergraduate research assistants.

-Conducted follow-up recruitment of participants.

\section{Massachusetts General Hospital, Boston, MA}

July 2007 - June 2010

Research Assistant/Coordinator. Supervisor: Keith Johnson, M.D.

-Prepared manuscripts, posters, graphs, and institutional review board amendments and reports.

-Obtained consent and administered neuropsychological testing for clinically normal, mild cognitively impaired, and Alzheimer's subjects.

-Created and maintained study databases. Analyzed image and psychological data. 


\section{Dartmouth Medical School, Lebanon, NH}

June 2004 - August 2006 (Summers)

Research Assistant. Supervisor: Karen Gillock, Ph.D.

-Recruited participants for posttraumatic stress disorder and depression studies.

-Scheduled and observed clinical interviews.

-Created study databases; compiled and analyzed interview data for each subject.

-Wrote and edited documents for publication and grant submission.

\section{RESEARCH INTERESTS}

Diagnostic methodology and treatment for dementia and cognitive decline. Awareness of cognitive decline among older adults.

Influence of age, environment, genetic factors, and attitude on cognitive ability.

\section{PUBLICATIONS}

Carmasin, J.S., Mast, B. T., Allaire, J.C., \& Whitfield, K.E. (2014). Vascular risk factors, depression, and cognitive change among African American older adults. International Journal of Geriatric Psychiatry, 29 (3), 291-8.

Johnson K.A., Sperling R.A., Gidicsin C.M., Carmasin J.S., Maye J.E., Coleman R.E., Reiman E.M., Sabbagh M.N., Sadowsky C.H., Fleisher A.S., Murali Doraiswamy P., Carpenter A.P., Clark C.M., Joshi A.D., Lu M., Grundman M., Mintun M.A., Pontecorvo M.J., \& Skovronsky D.M. (2013). Florbetapir (F18-AV-45) PET to assess amyloid burden in Alzheimer's disease dementia, mild cognitive impairment, and normal aging. Alzheimer's and Dementia, 9 (5 Suppl), S72-83.

Gurol M.E., Viswanathan A., Gidicsin C., Hedden, T., Martinez S.R., Dumas A., Vashkevich, A., Ayres A.M., Auriel, E., van Etten, E., Becker, A., Carmasin, J., Schwab, K., Rosand, J., Johnson, K.A., \& Greenberg, S.M. (2013). Cerebral amyloid angiopathy burden associated with leukoaraiosis: A PET/MRI study. Annals of Neurology, 73 (4), 529-36.

Amariglio, R. E., Becker, J. A., Carmasin, J., Wadsworth, L. P., Lorius, N., Sullivan, C., Maye, J., Gidicsin, C., Pepin, L. C., Sperling, R.A., Johnson, K.A., \& Rentz, D. M. (2012). Subjective cognitive complaints and amyloid burden in cognitively normal older individuals. Neuropsychologia, 50 (12), 2880-6. doi:

10.1016/j.neuropsychologia.2012.08.011

Gurol, M. E., Dierksen, G., Betensky, R.A., Gidicsin, C., Halpin, A., Becker, A., Carmasin, J., Ayres, A., Schwab, K., Viswanathan, A., Salat, D., Rosand, J., Johnson, K. A., \& Greenberg, S.M. (2012). Predicting sites of new hemorrhage with amyloid imaging in cerebral amyloid angiopathy. Neurology, 79 (4), 320-6. 
Rentz, D. M., Amariglio, R. E., Becker, J. A., Frey, M., Olson, L. E., Frishe, K., Carmasin, J., Maye, J.E., Johnson, K.A., \& Sperling, R.A. (2011). Face-name associative memory performance is related to amyloid burden in normal elderly. Neuropsychologia, 49(9), 2776-83.

Mast, B.T., Yochim, B., Carmasin, J.S., \& Rowe, S.V. (2011). Vascular depression: A neuropsychological perspective. In F. López-Muñoz and C. Álamo (Eds.), Neurobiology of depression (pp. 445-454). Boca Raton, FL: CRC Press.

Becker, J., Hedden, T., Carmasin, J., Maye, J., Rentz, D., Putcha, D., Fischl, B., Greve, D., Marshall, G., Salloway, S., Marks, D., Buckner, R., Sperling, R., \& Johnson, K. (2011). Amyloid-beta associated cortical thinning in clinically normal elderly. Annals of Neurology, 69 (6), 1032-42.

\section{POSTER PRESENTATIONS}

Carmasin, J.S., Mast, B.T., Allaire, J.C., \& Whitfield, K.E. (2012, November). Vascular burden, depression, and cognitive decline among African-American older adults. Poster presented at the Sixty-Fifth Annual Meeting of the Gerontological Society of America, San Diego, CA.

Carmasin, J., Rowe, S., \& Mast, B. (2011, November). Reliability and validity testing of the MARS-MFS among community-dwelling older adults. Poster presented at the SixtyFourth Annual Meeting of the Gerontological Society of America, Boston, MA.

Rowe, S., Shouse, J., Carmasin, J., \& Mast, B.T. (2011, February). Awareness of cognitive functioning and theory of mind in community-dwelling older adults. Poster presented at the Thirty-Ninth Annual Meeting of the International Neuropsychological Society, Boston, MA.

Carmasin, J., Maye, J., Marshall, G., Becker, J., Sperling, R., Rentz, D., \& Johnson, K. (2010, April). Overestimation of memory performance in normal elderly subjects is associated with amyloid burden in the temporal lobe. Poster presented at the Fourth International Human Amyloid Imaging Conference, Toronto, Canada.

Maye, J., Carmasin, J., Becker, J., Rentz, D., Sperling, R., \& Johnson, K. (2010, April). Maternal history of dementia is associated with amyloid deposition in clinically normal older individuals. Poster presented at the Fourth International Human Amyloid Imaging Conference, Toronto, Canada.

Becker, J., Carmasin, J., Maye, J., Rentz, D., Buckner, R., Sperling, R., \& Johnson, K. (2010, April). Amyloid deposition and FDG metabolism in relation to age in APOE4 carriers. Poster presented at the Fourth International Human Amyloid Imaging Conference, Toronto, Canada. 
Rentz, D., Becker, J., Frey, M., Carmasin, J., Maye, J., Olson, L., Johnson, K., \& Sperling, R. (2010, April). A challenging test of name retrieval may be sensitive to early amyloid deposition in normals. Poster presented at the Fourth International Human Amyloid Imaging Conference, Toronto, Canada.

Marshall, G., Carmasin, J., Hamdi, I., Frey, M., Olson, L., Rentz, D., Sperling, R., \& Johnson, K. (2009, July). Amyloid deposition and instrumental activities of daily living impairment in non-demented elderly. Poster presented at the International Conference on Alzheimer's Disease, Vienna, Austria.

Rentz, D., Locascio, J., Becker, J., Frey, M., Carmasin, J., Buckner, R., Sperling, R., \& Johnson, K. (2009, July). Cognitive reserve modifies the relationship of neuropsychological performance and amyloid deposition in normal elderly subjects. Poster presented at the International Conference on Alzheimer's Disease, Vienna, Austria.

Frey, M., Becker, J., Maye, J., Hedden, T., Carmasin, J., Olson, L., Mehta, A., Rastegar, S., Johnson, K., Buschke, H., Sperling, R., \& Rentz, D. (2009, July). Challenging tests of memory are more sensitive to detect effects of amyloid deposition in normal elderly subjects. Poster presented at the International Conference on Alzheimer's Disease, Vienna, Austria.

Sperling, R., Laviolette, P., O’Brien, J., O’Keefe, K., DeLuca, A., Carmasin, J., Becker, J., Pihlajamaki, M., Marshall, G., Rentz, D., Buckner, R., \& Johnson, K. (2008, November). Impaired default network fMRI activity is associated with parietal amyloid deposition in non-demented older individuals. Poster presented at the Thirty-Eighth Annual Meeting of the Society for Neuroscience, Washington, DC.

Becker, J., Carmasin, J., Fischl, B., Greve, D., DeLuca, A., LaViolette, P., Fischman, A., Rentz, D., Sperling, R., \& Johnson, K. (2008, June). Amyloid deposition and cortical thickness in aging and Alzheimer's disease. Poster presented at the Society of Nuclear Medicine, New Orleans, LA.

Marshall, G., Carmasin, J., Moran, E., Eng, E., Binstock, A., Becker, J., Fischman, A., Rentz, D., Sperling, R., \& Johnson, K. (2008, April). PiB uptake in non-demented elderly Apolipoprotein E4 carriers. Poster presented at the American Academy of Neurology, Chicago, IL.

Rentz, D., Carmasin, J., Eng, E., Becker, J., Sperling, R., \& Johnson, K. (2008, April). Cognitive change in non-demented subjects with amyloid deposition. Poster presented at the Second International Human Amyloid Imaging Conference, Chicago, IL. 